\title{
ON THE STABILITY OF THE FEASIBLE SET IN OPTIMIZATION PROBLEMS*
}

\author{
N. DINH ${ }^{\dagger}$, M.A. GOBERNA ${ }^{\ddagger}$, AND M.A. LÓPEZ
}

\begin{abstract}
This paper provides stability theorems for the feasible set of optimization problems posed in locally convex topological vector spaces. The problems considered in this paper have an arbitrary number of inequality constraints and one constraint set. Different models are discussed, depending on the properties of the constraint functions (linear or not, convex or not, but at least lower semicontinuous) and one closed constraint set (but not necessarily convex). The parameter space is formed by systems of the same type as the nominal one (with the same space of variables and the same number of constraints), where the constraint set can be perturbed or not, equipped with the metric of the uniform convergence on the positive multiples of a fixed barrelled neighborhood of zero. In finite dimensions, this topology describes the unifom convergence on compact sets and, in the particular case that the constraints are linear, the uniform convergence of the vector coefficients. The paper examines, in a unified way, the lower and upper semicontinuity, and the closedness, of the feasible set mapping, the stable consistency of the constraint system with respect to arbitrary and right-hand side perturbations, Tuy and Robinson regularities, and other desirable stability properties of the feasible set.
\end{abstract}

Key words. feasible set, stability, infinite optimization, semi-infinite optimization

AMS subject classification. 90C $31,90 \mathrm{C} 48,90 \mathrm{C} 34,49 \mathrm{~K} 40$

1. Introduction. Many optimization problems are formulated in the form

$$
\begin{aligned}
\text { (P) inf } & f(x) \\
\text { s.t. } & f_{t}(x) \leq 0, \forall t \in T ; \\
& x \in C,
\end{aligned}
$$

where $T$ is an arbitrary (possibly infinite, possibly empty) index set, $C \subset X$ is the constraint set, the decision space $X$ is a locally convex Hausdorff topological vector space (possibly $\mathbb{R}^{n}$ ), and the constraint functions $f_{t}$ are extended, i.e., $f_{t}: X \rightarrow$ $\mathbb{R} \cup\{+\infty\}$ for all $t \in T$. In this paper we analyze the stability of the feasible set of $(\mathrm{P})$, say $F$, under several types of perturbations of the data preserving the decision space $X$ and the index set $T$.

The main questions regarding the stability of the feasible set in optimization problems were already posed in 1975 by S.M. Robinson: "What happens to the solution set when the data are subject to small perturbations? In particular, will the perturbed system be solvable? If so, will the solution set change gradually?" ([46], where $X$ is a Banach space and $f_{t}$ is affine for all $t \in T$ ). Answering these questions, it is possible to extend this analysis to other relevant elements of the problem: "If a mathematical program lacks continuity, then small changes in parameters or functions (often due to inexact estimates of the parameters or functions) may result in large changes in the optimal solutions or in the optimal objective function values or both. Another possibly even more important need for continuity in mathematical programs is the fact that digital computers operate with finite arithmetic and often produce significant roundoff errors over time. Continuity of the mathematical program being

\footnotetext{
*This research was partially supported by MICINN of Spain, Grant MTM2008-06695-C03-01.

${ }^{\dagger}$ Department of Mathematics, International University, Vietnam National University-HCM city, Linh Trung ward, Thu Duc district, Ho Chi Minh city, Vietnam.

${ }^{\ddagger}$ Department of Statistics and Operations Research, University of Alicante, 03080 Alicante, Spain.

$\S^{\S}$ Department of Statistics and Operations Research, University of Alicante, 03080 Alicante, Spain.
} 
solved gives credence to the belief that the algorithmic process being used may lead to an optimal or near-optimal solution of the problem. Lack of continuity, on the other hand, could mean that the algorithm is yielding something far from optimal" ([23], where $X=\mathbb{R}^{n}$ and $T$ is arbitrary). In [12] the authors emphasize the need of stability analysis of the feasible set for an infinite-dimensional optimization problem arising in the optimal control of a system of $n$ water reservoirs $R_{1}, R_{2}, \ldots, R_{n}$. The model is based on the realistic assumption that if more raining water flows into the reservoirs that they can hold, the rest can be sold to a neighboring dry region, provided that the demand of the region is satisfied. Conversely, if the inflows are short, and the reservoirs have free capability for additional water, some water can be bought from outside to meet the inner demand. In this problem a set of decision variables are the rates $x_{i}(t)$ at which water is fed from $R_{i}$ at time (we assume that $x_{i}, i=1,2, \ldots, n$, are continuous functions in the operating interval of time $[a, b])$, and a second group of variables $y_{i}, i=1,2, \ldots, n$, provides the "selling" rate of water from $R_{i}$ at $t$, which is given by $d y_{i}(t)$ (now it makes sense to require that functions $y_{i}, i=1,2, \ldots, n$, are of bounded variation, since these planned inflows $\left(d y_{i}(t)>0\right)$ or outflows $\left(d y_{i}(t)<0\right)$ take place in punctual instants of time in $[a, b])$. Constraints come from the need of satisfying the overall demand at each instant $t \in[a, b]$, and not exceeding the capability of each reservoir along all the operating time. Since the raining inflows and demand are necessarily uncertain, it is a crucial issue to study the stability of this problem with respect to perturbations of the uncertain data.

In this paper, we consider the effect on the solution set of the constraint system

$$
\sigma:=\left\{f_{t}(x) \leq 0, t \in T ; x \in C\right\}
$$

also represented by its corresponding data set, $\left\{f_{t}, t \in T ; C\right\}$, of perturbing any constraint function $f_{t}, t \in T$, and possibly the constraint set $C$, under the condition that these perturbations preserve certain properties of the constraints. In particular, we analyze the continuity properties (in the sense of [?], [4] or [47]) of the feasible set mapping associating to each perturbed system its corresponding solution set. The parameter space, generically denoted by $\Theta$, is a given family of systems with the same decision space and index set as $\sigma$, satisfying its relevant properties and such that

$$
\sigma_{1}=\left\{f_{t}^{1}, t \in T ; C_{1}\right\} \in \Theta \Rightarrow\left\{f_{t}^{1}+\alpha, t \in T ; C_{1}\right\} \in \Theta \forall \alpha \in \mathbb{R}
$$

(i.e., closedness of $\Theta$ with respect to the addition of a fixed constant to the constraint functions) if $T \neq \emptyset$. The corresponding feasible set mapping is $\mathcal{F}: \Theta \rightrightarrows X$ such that

$$
\begin{aligned}
\mathcal{F}\left(\sigma_{1}\right) & =\left\{x \in X: f_{t}^{1}(x) \leq 0, \forall t \in T ; x \in C_{1}\right\} \\
& =\left\{x \in X: g^{1}(x) \leq 0 ; x \in C_{1}\right\}, \text { if } T \neq \emptyset,
\end{aligned}
$$

where $g^{1}:=\sup _{t \in T} f_{t}^{1}$ denotes the marginal function of $\sigma_{1}$.

Observe that changes in the representation of $F=\mathcal{F}(\sigma)$ could provoke changes in the parameter space (e.g., the aggregation of nonnegative linear combinations of the functional constraints in $\sigma$ could enlarge the index set although it does not affect the solution set). This paper considers seven main parameter spaces, namely 


$$
\begin{aligned}
& \Theta_{1}:=\left\{\left\{f_{t}^{1}, t \in T ; C_{1}\right\}: f_{t}^{1}: X \rightarrow \mathbb{R} \cup\{+\infty\} \forall t \in T \text { and } \emptyset \neq C_{1} \subset X\right\}, \\
& \Theta_{2}:=\left\{\sigma_{1} \in \Theta_{1}: f_{t}^{1} \text { is lsc } \forall t \in T \text { and } C_{1} \text { is closed }\right\}, \\
& \Theta_{3}:=\left\{\sigma_{1} \in \Theta_{2}: \text { the local minima of } g^{1} \text { are global, } C_{1}\right. \text { is convex, } \\
& \text { and } \left.\mathcal{F}\left(\sigma_{1}\right) \subset \operatorname{int} C_{1}\right\}, \\
& \Theta_{4}:=\left\{\sigma_{1} \in \Theta_{2}: \text { the local minima of } g^{1} \text { are global and } C_{1}=X\right\}, \\
& \Theta_{5}:=\left\{\sigma_{1} \in \Theta_{2}: f_{t}^{1} \text { convex } \forall t \in T \text { and } C_{1} \text { is convex }\right\}, \\
& \Theta_{6}:=\left\{\sigma_{1} \in \Theta_{5}: f_{t}^{1} \text { is finite-valued } \forall t \in T \text { and } C_{1}=C_{0}\right\}, \text { and } \\
& \Theta_{7}:=\left\{\sigma_{1} \in \Theta_{2}: f_{t}^{1}=u_{t}+\alpha_{t},\left(u_{t}, \alpha_{t}\right) \in X^{*} \times \mathbb{R} \forall t \in T, \text { and } C_{1}=X\right\},
\end{aligned}
$$

where lsc stands for lower semicontinuous, $C_{0}$ is a fixed closed convex subset of $X$ (e.g., the whole space $X$ or the solution set of the subsystem of nonperturbable constraints, which could include equations, sign constraints, etc.), and $X^{*}$ denotes the topological dual of $X$. The above parameter spaces are related by inclusion as the following diagram shows:

$$
\begin{array}{lllllll}
\Theta_{4} & \subset & \Theta_{3} & \subset & \Theta_{2} & \subset & \Theta_{1} \\
\Theta_{7} & \subset & \Theta_{6} & \subset & \Theta_{5} & &
\end{array}
$$

Observe that $\Theta_{1}, \Theta_{2}, \Theta_{5}, \Theta_{6}$, and $\Theta_{7}$ are closed with respect to (w.r.t.) perturbations of the right-hand side (RHS), i.e., replacing 0 by (possibly different) scalars in each constraint. Concerning $\Theta_{3}$, the functions whose local minima are global have been characterized in [50] in terms of the lower semicontinuity of the feasible set mapping corresponding to $\left\{x \in \mathbb{R}^{n}: g(x) \leq 0\right\} \in \Theta_{3}$ (with a unique index) w.r.t. the righthand side (RHS) scalar, in [36] in terms of generalized convexity, and in [26] in terms of arcwise quasiconvexity (the first two papers with $X=\mathbb{R}^{n}$ and the 3 rd one with $X$ being a metric space). A class of functionals arising in control problems that enjoy this local-global property has been identified in [5]. The next simple example shows a significant element of $\Theta_{4} \backslash \Theta_{5}$ with $X=\mathbb{R}^{n}$ and $T \neq \emptyset$ arbitrary.

ExAmple 1.1. Let $\sigma=\left\{f_{t}, t \in T ; \mathbb{R}^{n}\right\}$ be such that $f_{t}(x)=\left\langle a_{t}, x\right\rangle-b_{t}$, where $a_{t}=\left(a_{t 1}, \ldots, a_{t n}\right) \in \mathbb{R}_{+}^{n} \backslash\left\{0_{n}\right\}$ and $b_{t} \in \mathbb{R}, t \in T$, and $\left\langle a_{t}, x\right\rangle:=\min _{i \in I_{+}\left(a_{t}\right)} a_{t i}\left|x_{i}\right|$, where $I_{+}\left(a_{t}\right):=\left\{i \in\{1, \ldots, n\}: a_{t i}>0\right\}$. In this case, the restriction of the marginal function of $\sigma, g$, to $\mathbb{R}_{+}^{n}$ is an lsc ICAR (acrostic of "increasing and convex along rays") function. Then, according to [48], $g$ satisfies the following two properties:

(i) Given $x, y \in \mathbb{R}^{n}$, if $\left|x_{i}\right| \geq\left|y_{i}\right|$ for all $i=1, \ldots, n$, then $g(x) \geq g(y)$.

(ii) Given $x \in \mathbb{R}^{n} \backslash\left\{0_{n}\right\}$, the function $g_{x}: \mathbb{R}_{+} \mapsto \mathbb{R}_{+}$such that $g_{x}(\lambda)=g(\lambda x)$ is convex.

Now we prove that the local minima of $g$ are global. In fact, (i) implies that $0_{n}$ is a global minimum. Thus we must show that $g(\bar{x})=g\left(0_{n}\right)$ for any local minimum $\bar{x}$. Otherwise, if $g(\bar{x})>g\left(0_{n}\right)$ and $\lambda \in[0,1[$, (ii) yields

$$
\begin{aligned}
g(\lambda \bar{x}) & =g_{\bar{x}}((1-\lambda) 0+\lambda 1) \leq(1-\lambda) g_{\bar{x}}(0)+\lambda g_{\bar{x}}(1) \\
& =(1-\lambda) g\left(0_{n}\right)+\lambda g(\bar{x})=g(\bar{x})-(1-\lambda)\left[g(\bar{x})-g\left(0_{n}\right)\right]<g(\bar{x}),
\end{aligned}
$$


and taking $\lambda \nearrow 1$ we conclude that $\bar{x}$ is not a local minimum.

When the parameter space of a given MP problem does not appear in the above list, it is usually easy to build up its corresponding stability theory by adapting the relative to some close space in the above list. In particular, if $\Theta_{j+1} \subset \Theta \subset \Theta_{j}$, any sufficient condition for the feasible set mapping corresponding to $\Theta_{j}$, say $\mathcal{F}_{j}$, to be closed (lsc, usc) at $\sigma \in \Theta_{j}$ guaranties that $\mathcal{F}$ is closed (lsc, usc) at $\sigma \in \Theta$. Analogously, any necessary condition for $\mathcal{F}_{j+1}$ to be closed (lsc, usc) at $\sigma \in \Theta_{j+1}$ is also necessary for $\mathcal{F}$ to be closed (lsc, usc) at $\sigma \in \Theta$. In this paper we analyze the continuity properties of the feasible set mapping $\mathcal{F}$ at the nominal system $\sigma$ relative to arbitrary perturbations of the constraint functions or just the RHS function (replacing the null function with certain $u \in \mathbb{R}^{T}$ ) whereas the constraint set will remain fixed or not in the different models. To do so we endow the basic space of parameters $\Theta_{1}$ with a suitable topology and consider $\Theta \subset \Theta_{1}$ equipped with the induced topology.

The closest antecedents of our study are [2] and [3], about $\mathcal{F}_{7}$ with $C_{0} \subset \mathbb{R}^{n}$ possibly nonclosed and nonconvex (linear semi-infinite systems with set constraint), [20], [21] and [19, Chapter 6], about $\mathcal{F}_{7}$ with $C_{0}=X=\mathbb{R}^{n}$ (linear semi-infinite systems). A particular case was first studied during the 1980s: [8] and [16] considered $\Theta \subset \Theta_{7}$ formed by linear systems (called continuous) $\left\{u_{t}(x)+\alpha_{t} \leq 0, t \in T\right\}$ such that $T$ is a compact Hausdorff space and the functions $u_{(\cdot)}: T \rightarrow \mathbb{R}^{n}$ and $\alpha_{(\cdot)}: T \rightarrow \mathbb{R}$ are continuous on $T$ (this study was later completed in [19, Chapter 6], where it was shown that the behavior of $\mathcal{F}$ for continuous semi-infinite linear systems and for general linear semi-infinite systems is quite similar, despite that perturbations are restricted to be continuous functions). Other antecedents are [42], about $\mathcal{F}_{7}$ with $C_{0}=X$ (linear infinite systems), and [44], about $\mathcal{F}_{6}$ with $C_{0}=X=\mathbb{R}^{n}$ (convex semi-infinite systems). In [42] the decision space is $X=Y^{*}$, where $Y$ is some metrizable locally convex Hausdorff topological vector space, so that $X^{*}$ can be identified with $Y$ if $X^{*}$ is endowed with the weak* topology, i.e., the parameter space is $\Theta_{7}$. [20], [21], and [44] provided the fundamentals for the stability analysis of the optimal set mapping and the optimal value function in linear and convex semi-infinite optimization from the same set-valued perspective (see [19, Chapter 10] and [17], respectively). The parameter space of the so-called min-type semi-infinite systems, whose constraint functions are the restriction to $C_{0}=X=\mathbb{R}_{++}^{n}$ of the constraint functions $f_{t}$ of Example 1.1, with $a_{t} \in \mathbb{R}_{++}^{n}$, is then close to $\Theta_{3}$ and its stability theory ([43]) is almost identical to the one of $\mathcal{F}_{3}$ developed in this paper (in this case $X$ is not a linear space). All the data defining $\sigma$ (except the constraint set) were considered perturbable in the mentioned papers, whereas in [14] we characterized the lower semicontinuity and the subdifferentiability at $\sigma \in \Theta_{5}$ of the optimal value function under perturbations of the RHS function 0 (i.e., the stability perspective of [35]). Let us mention that, in the finite dimensional setting, with fixed constraint set $C_{0}=\mathbb{R}^{n}$, there exists a third stability perspective, consisting of introducing a parametrization mapping describing particular types of perturbations of $(\mathrm{P})$. Under suitable smoothness assumptions on these mappings it is possible to obtain strong topological properties of the feasible set and the optimal set mappings, as well as the geometrical analysis of the trajectory described by the optimal solution, if it unique (see, e.g., [30] and [32]). In particular, the so-called extended Mangasarian-Fromovitz constraint qualification (EMFCQ, in brief) was introduced in the semi-infinite setting in [32] inspired in a condition given in [24] (see [31] for a parametric counterpart). In linear semi-infinite optimization, the relationship between EMFCQ, the Robinson c.q., and the metric regularity of the feasible set mapping is explored in [9] and 
[10]. [33] is focused on the study of metric regularity in connection with EMFCQ of certain parametrized nonlinear semi-infinite systems with $C^{1}$-data and subject to RHS perturbations. The finite dimension of the decision space seems to be substantial in this kind of stability analysis. The stability theory of the feasible set in semiinfinite programming has been reviewed in [18], where the connection between lower semicontinuity of $\mathcal{F}$ and constraint qualifications (e.g., Slater-type and interior-type conditions) are discussed. There exists a wide literature on constraint qualifications in convex (and extended convex) infinite dimensional optimization, where they provide optimality conditions and duality theorems (see, e.g., [6], [15], [28], [29], [36], [38], [39], [40], and [41]).

The paper is organized as follows. $§ 2$ introduces most stability concepts considered in this paper. Among the stability concepts left aside in our study, let us mention those related with the dimension of $F$ (dimensional stability and topological stability) and the metric regularity of the inverse mapping $\mathcal{F}^{-1}$, a theory still in progress in the linear semi-infinite context (see, e.g., [19], [9], and references therein). $\S 3$ introduces a metric on any parameter space $\Theta \subset \Theta_{1}$, and shows that $\Theta_{j}$ is complete if $j=1,2,5,6,7$. The definition of such a metric is inspired in [44] although the size of the perturbation of an individual function is defined here in a slightly different way and a suitable measure of the perturbation of the constraint set has also been introduced. The completeness of $\Theta$ could be useful in order to characterize the metric regularity of $\mathcal{F}^{-1}$ by adapting powerful results on metric regularity in Banach spaces (e.g., [27] and references therein). $\S 4$ shows that $\mathcal{F}$ is closed on any $\Theta \subset \Theta_{2}$. The main result in $\S 5$ is Theorem 5.1, which characterizes the lower semicontinuity of $\mathcal{F}$ at a given $\sigma \in \Theta$ when either $\Theta \subset \Theta_{3}$ or $\Theta \subset \Theta_{5}$ by means of conditions (ii)-(vi) or conditions (ii)-(viii), respectively. All the results in $\S 3-\S 5$ are valid, then, for normable spaces. In the last two sections $X$ is either a normable space or certain type of topological space with no linear structure. In $\S 6$, we adapt the concept of Robinson regularity to systems posed on a normable space $X$, and we characterize this property for $\Theta \subset \Theta_{5}$. Finally, in $\S 7$, we characterize the upper semicontinuity of $\mathcal{F}: \Theta \rightrightarrows X$ when either $X=\mathbb{R}^{n}$ or $X$ is a metrizable locally compact and sigma-compact (i.e., union of a countable family of compact sets) space $X$, with $\Theta$ subspace of $\Theta_{2}$ or some space of systems with continuous constraints, respectively. Recall that the decision space in [43], $X=\mathbb{R}_{++}^{n}$, is a metric locally compact and sigma-compact topological space.

The main novelty of this paper in comparison with the previous ones is the key role played here by the parameter space, which allows us to give very general results (showing, for instance, that the closedness of the feasible set mapping only requires lower semicontinuity of the constraint function and closedness of the constraint set) and, from a methodological perspective, the use of infinite dimensional convex analysis, nets (instead of sequences) and linear representations of $F$ involving epigraphs of the conjugates of the constraint functions (instead of their subddiferentials, which could not exist in our general framework).

2. Preliminaries. The dual space of $X$ is denoted by $X^{*}$. For a set $D \subset X$, we denote with conv $D$ and cone $D$ the convex hull of $D$ and the convex conical hull of $D \cup$ $\{0\}$, respectively. If $D=\left\{d_{s}, s \in S\right\}$, denoting by $\mathbb{R}^{(S)}$ the linear space of mappings from $S$ to $\mathbb{R}$ with finite support and by $\mathbb{R}_{+}^{(S)}$ its positive cone, we can write cone $D=$ $\left\{\sum_{s \in S} \lambda_{s} d_{s}: \lambda \in \mathbb{R}_{+}^{(S)}\right\}$ and conv $D=\left\{\sum_{s \in S} \lambda_{s} d_{s}: \lambda \in \mathbb{R}_{+}^{(S)}, \sum_{s \in S} \lambda_{s}=1\right\}$. From the topological side, we denote by $\mathcal{N}(x)$ the family of all the neighborhoods of $x \in X$ and by $\operatorname{cl} D$ the closure of $D$, if $D \subset X$, and the closure of $D$ w.r.t. the weak* 
topology, if $D \subset X^{*} \times \mathbb{R}$. The indicator function $\delta_{D}$ is defined as $\delta_{D}(x)=0$ if $x \in D$, and $\delta_{D}(x)=+\infty$ if $x \notin D$. D is a nonempty closed convex set if and only if $\delta_{D}$ is a proper lsc convex function.

Now let $h: X \rightarrow \mathbb{R} \cup\{+\infty\}$. The effective domain, the graph, and the epigraph of $h$ are $\operatorname{dom} h=\{x \in X: h(x)<+\infty\}$, gph $h=\{(x, \gamma) \in X \times \mathbb{R}: h(x)=\gamma\}$, and epi $h=\operatorname{gph} h+\operatorname{cone}\{(0,1)\}$ (with the convention that $A+\emptyset=\emptyset+A=\emptyset$ ), respectively, whereas the conjugate function of $h, h^{*}: X^{*} \rightarrow \mathbb{R} \cup\{ \pm \infty\}$, is defined by

$$
h^{*}(v)=\sup \{\langle v, x\rangle-h(x): x \in \operatorname{dom} h\} .
$$

It is well-known that, if $h$ is a proper lsc convex function, then $h^{*}$ enjoys the same properties and its conjugate, denoted by $h^{* *}: X \rightarrow \mathbb{R} \cup\{ \pm \infty\}$, defined by

$$
h^{*}(x)=\sup \left\{\langle v, x\rangle-h^{*}(v): v \in \operatorname{dom} h^{*}\right\}
$$

coincides with $h . \delta_{C}^{*}$ is the support function of $C$, whose epigraph epi $\delta_{C}^{*}$ is a closed convex cone.

If $\left\{f_{t}, t \in T\right\}$ is a family of proper convex lsc functions such that $\operatorname{dom}\left(\sup _{t \in T} f_{t}\right) \neq$ $\emptyset$, one has that

$$
\operatorname{epi}\left(\sup _{t \in T} f_{t}\right)^{*}=\operatorname{clconv}\left(\bigcup_{t \in T} \operatorname{epi} f_{t}^{*}\right)
$$

(see, e.g., [7], [40], and [41]).

Let $\sigma=\left\{f_{t}(x) \leq 0, t \in T ; x \in C\right\}$ be consistent and let $v \in X^{*}$ and $\alpha \in \mathbb{R}$. Then the asymptotic Farkas' Lemma (Theorem 4.1 in [13]) establishes that

$$
f_{t}(x) \leq 0 \forall t \in T, x \in C \Longrightarrow v(x) \leq \alpha
$$

if and only if

$$
(v, \alpha) \in \operatorname{clcone}\left(\bigcup_{t \in T} \operatorname{epi} f_{t}^{*} \cup \operatorname{epi} \delta_{C}^{*}\right) .
$$

From the separation theorem, (2.2), and the equation

$$
\operatorname{clcone}\left(\bigcup_{t \in T} \operatorname{epi} f_{t}^{*} \cup \operatorname{epi} \delta_{C}^{*}\right)=\operatorname{clcone}\left\{\left(\bigcup_{t \in T} \operatorname{epi} f_{t}^{*}\right)+\operatorname{epi} \delta_{C}^{*}\right\},
$$

when $T \neq \emptyset([13])$ we get the following linear representations of $F$ (i.e., linear systems whose solution set is $F)$ :

$$
\left\{v(x) \leq \alpha,(v, \alpha) \in\left(\bigcup_{t \in T} \text { epi } f_{t}^{*}\right)+\operatorname{epi} \delta_{C}^{*} ; x \in X\right\}, \text { if } T \neq \emptyset,
$$

and

$$
\left\{v(x) \leq \alpha,(v, \alpha) \in \bigcup_{t \in T} \operatorname{epi} f_{t}^{*} \cup \operatorname{epi} \delta_{C}^{*} ; x \in X\right\}
$$


Observing that $v(x) \leq \alpha$ if and only if $v(x)-\frac{1}{k} \leq \alpha$ for all $k \in \mathbb{N}$ if and only if $k v(x) \leq k \alpha+1$ for all $k \in \mathbb{N}$, we can replace in (2.4) epi $\delta_{C}^{*}$ with

$$
\left[\bigcup_{k \in \mathbb{N}}\left(k \text { epi } \delta_{C}^{*}\right)\right]+(0,1)=\left(\operatorname{epi} \delta_{C}^{*}\right)+(0,1) .
$$

Thus

$$
\left\{v(x) \leq \alpha,(v, \alpha) \in \bigcup_{t \in T} \operatorname{epi} f_{t}^{*} \cup\left[\left(\operatorname{epi} \delta_{C}^{*}\right)+(0,1)\right] ; x \in X\right\} .
$$

is another linear representation of $F$.

Let $\left\{A_{\delta}\right\}_{\delta \in \Delta}$ be a net of subsets of $X$ associated with the directed set $(\Delta, \preceq)$. We define the set of limit points of this net as the set

$$
\begin{aligned}
\operatorname{Li}_{\delta} A_{\delta} & =\left\{\begin{array}{l|l}
x \in X & \begin{array}{l}
\text { for all } U \in \mathcal{N}(x) \text { there exists } \delta \in \Delta \text { such that } \\
U \cap A_{\delta^{\prime}} \neq \emptyset \text { for every } \delta^{\prime} \in \Delta \text { such that } \delta \preceq \delta^{\prime}
\end{array}
\end{array}\right\} \\
& =\left\{\begin{array}{l|l}
x \in X & \begin{array}{l}
\text { for all } U \in \mathcal{N}(x), U \text { intersects } A_{\delta} \\
\text { for all } \delta \text { in some residual subset of } \Delta
\end{array}
\end{array}\right\},
\end{aligned}
$$

and the set of cluster points of the net as the set

$$
\begin{aligned}
\underset{\delta}{\operatorname{Ls}} A_{\delta} & =\left\{\begin{array}{l|l}
x \in X & \begin{array}{l}
\text { for all } U \in \mathcal{N}(x) \text { and for every } \delta \in \Delta \text { there } \\
\text { exists } \delta^{\prime} \in \Delta \text { such that } \delta \preceq \delta^{\prime} \text { and } U \cap A_{\delta^{\prime}} \neq \emptyset
\end{array}
\end{array}\right\} \\
& =\left\{\begin{array}{l|l}
x \in X & \begin{array}{l}
\text { for all } U \in \mathcal{N}(x), U \text { intersects } A_{\delta} \\
\text { for all } \delta \text { in some cofinal subset of } \Delta
\end{array}
\end{array}\right\} .
\end{aligned}
$$

Clearly $\operatorname{Li}_{\delta} A_{\delta} \subset \operatorname{Ls}_{\delta} A_{\delta}$ and both sets are closed, whether or not the terms of the net are closed. We say that $\left\{A_{\delta}\right\}_{\delta \in \Delta}$ converges in the sense of Kuratowski-Painlevé to the closed set $A$ if $\operatorname{Li}_{\delta} A_{\delta}=\operatorname{Ls}_{\delta} A_{\delta}=A$.

The domain of the feasible set mapping $\mathcal{F}: \Theta \rightrightarrows X$, where $\Theta$ is some space of parameters equipped with the metric defined in (3.5), is $\operatorname{dom} \mathcal{F}=\{\sigma \in \Theta: \mathcal{F}(\sigma) \neq \emptyset\}$. Obviously, if $\sigma=\left\{f_{t}, t \in T ; C\right\} \in \operatorname{dom} \mathcal{F}$, then $f_{t}$ is proper for all $t \in T$. The main objective of this paper is the characterization of the following (local or global) desirable properties of $\mathcal{F}$, which adapt to our general framework similar ones appeared in the works mentioned in $\S 1$.

$\mathcal{F}$ is closed at $\sigma=\left\{f_{t}, t \in T ; C\right\} \in \Theta$ if for all nets $\left\{\sigma_{\delta}\right\}_{\delta \in \Delta} \subset \Theta$ and $\left\{x_{\delta}\right\}_{\delta \in \Delta} \subset$ $X$ satisfying $x_{\delta} \in \mathcal{F}\left(\sigma_{\delta}\right)$ for all $\delta \in \Delta, \lim _{\delta} \sigma_{\delta}=\sigma$ and $\lim _{\delta} x_{\delta}=x$, one has $x \in \mathcal{F}(\sigma)$. $\mathcal{F}$ is said to be closed if it is closed at $\sigma$ for all $\sigma \in \Theta$. Obviously, $\mathcal{F}$ is closed if and only if its graph, gph $\mathcal{F}:=\{(\sigma, x) \in \Theta \times X: x \in \mathcal{F}(\sigma)\}$, is a closed set in the product space.

$\mathcal{F}$ is lower semicontinuous at $\sigma \in \Theta$ in the Kuratowski-Berge sense (lsc, in brief) if, for each open set $W \subset X$ such that $W \cap \mathcal{F}(\sigma) \neq \emptyset$, there exists an open set $V \subset \Theta$, containing $\sigma$, such that $W \cap \mathcal{F}\left(\sigma_{1}\right) \neq \emptyset$ for each $\sigma_{1} \in V$. $\mathcal{F}$ is said to be lsc if it is lsc at $\sigma$ for all $\sigma_{1} \in \Theta$.

$\mathcal{F}$ is upper semicontinuous at $\sigma \in \Theta$ in the Kuratowski-Berge sense (usc, in brief) if, for each open set $W \subset X$ such that $\mathcal{F}(\sigma) \subset W$, there exists an open set $V \subset \Theta$, containing $\sigma$, such that $\mathcal{F}\left(\sigma_{1}\right) \subset W$ for each $\sigma_{1} \in V$.

We say that $\sigma$ satisfies the strong Slater condition if there exists some $\bar{x} \in C$ and some $\rho>0$ such that $f_{t}(\bar{x})<-\rho$ for all $t \in T$ (i.e., $g(\bar{x})<-\rho$ ). In such a case, $\bar{x}$ is called strong Slater (SS) point of $\sigma$ with associated constant $\rho$. 
Moreover, we say that $\sigma$ is Tuy regular if there exists $\epsilon>0$ such that for any $w \in \mathbb{R}^{T}$ and any nonempty convex set $C_{1} \subset X$ satisfying $\sigma_{1}:=\left\{f_{t}(x)-w_{t} \leq 0, t \in\right.$ $\left.T ; x \in C_{1}\right\} \in \Theta$ and $\max \left\{\sup _{t \in T}\left|w_{t}\right|, d\left(\delta_{C}, \delta_{C_{1}}\right)\right\}<\epsilon$, where $d\left(\delta_{C}, \delta_{C_{1}}\right)$ is given by $(3.2)$, one has $\mathcal{F}\left(\sigma_{1}\right) \neq \emptyset$. This desirable property inspired in [49] means that sufficiently small perturbations of the RHS scalars and the constraint set preserve the consistency of the nominal system.

Other stability concepts are introduced in $\S 5$ and $\S 6$.

3. The parameter spaces. In order to define a suitable topology on the parameter space $\Theta$ we introduce, first, the distance between two extended functions in the sense of the uniform convergence on the positive multiples of the closure of a fixed barrelled neighborhood of zero, say $B$, and second, from this distance, another one between inequality systems posed in $X$ and indexed with $T$. Obviously, the sets $B_{k}:=k B, k \in \mathbb{N}$, are also barrelled neighborhoods of zero such that $\bigcup_{k \in \mathbb{N}} B_{k}=X$. Since $B_{k} \subset \operatorname{int} B_{k+1}$ for all $k$, we have also $\bigcup_{k \in \mathbb{N}} \operatorname{int} B_{k}=X$. If $X$ is normable, we shall take as $B$ a bounded barrel (see, for instance, $[25, \S 10 \mathrm{C}]$ ).

Let $\mathcal{V}_{1}$ be the set of all functions of the form $f: X \rightarrow \mathbb{R} \cup\{+\infty\}$. For each pair of functions $f, h \in \mathcal{V}_{1}$, we define

$$
\begin{aligned}
d_{k}(f, h) & :=\sup _{x \in B_{k}}|f(x)-h(x)|, k \in \mathbb{N} ; \\
d(f, h) & :=\sum_{k=1}^{+\infty} 2^{-k} \min \left\{1, d_{k}(f, h)\right\} .
\end{aligned}
$$

Here, by convention, we understand that $(+\infty)-(+\infty)=0,|-\infty|=+\infty,|+\infty|=$ $+\infty$.

It is worth noting that $d(f, h)=0$ implies that, for any $k \in \mathbb{N},|f(x)-h(x)|=0$ for all $x \in B_{k}$. By our convention, either $f(x)=h(x)=+\infty$ or $f(x)=h(x) \in \mathbb{R}$. As $\bigcup_{k} B_{k}=X, f(x)=h(x)$ for all $x \in X$. Moreover, it is easy to verify that $\left(\mathcal{V}_{1}, d\right)$ is a metric space.

Observe that, given a nonempty set $C_{1} \subset X, C_{1} \neq C$, if

$$
k_{1}:=\min \left\{k \in \mathbb{N}: B_{k} \cap C \neq B_{k} \cap C_{1}\right\}
$$

then

$$
d\left(\delta_{C}, \delta_{C_{1}}\right)=2^{1-k_{1}} .
$$

LEMma 3.1. Let $k \in \mathbb{N}$ and $\epsilon>0$ be given. There exists $\rho>0$ such that $d_{k}(f, h)<\epsilon$ for each pair $f, h \in \mathcal{V}_{1}$ satisfying $d(f, h)<\rho$.

Proof. Let $\epsilon \in(0,1)$. Take $\rho>0$ such that $\rho<2^{-k} \epsilon$. If $f, h \in \mathcal{V}_{1}$ then

$$
\begin{aligned}
d(f, h)<\rho & \Rightarrow 2^{-k} \min \left\{1, d_{k}(f, h)\right\}<2^{-k} \epsilon \\
& \Rightarrow \min \left\{1, d_{k}(f, h)\right\}<\epsilon \\
& \Rightarrow d_{k}(f, h)<\epsilon .
\end{aligned}
$$

LEMMA 3.2. For each $\epsilon>0$, there exist $k \in \mathbb{N}$ and $\rho>0$ such that $d(f, h)<\epsilon$ for each pair $f, h \in \mathcal{V}_{1}$ satisfying $d_{k}(f, h)<\rho$. 
Proof. Let $\epsilon>0$. Take $k \in \mathbb{N}$ such that $\sum_{i=k+1}^{+\infty} 2^{-i}<\frac{\epsilon}{2}$. Choose $\left.\rho \in\right] 0,1[$ satisfying $\rho \sum_{i=1}^{k} 2^{-i}<\frac{\epsilon}{2}$.

Note that $d_{i}(f, h) \leq d_{k}(f, h)$ whenever $i \leq k$. If $d_{k}(f, h)<\rho$, then

$$
\begin{aligned}
d(f, h) & =\sum_{i=1}^{k} 2^{-i} \min \left\{1, d_{i}(f, h)\right\}+\sum_{i=k+1}^{+\infty} 2^{-i} \min \left\{1, d_{i}(f, h)\right\} \\
& \leq \sum_{i=1}^{k} 2^{-i} \rho+\sum_{i=k+1}^{+\infty} 2^{-i}<\frac{\epsilon}{2}+\frac{\epsilon}{2}=\epsilon .
\end{aligned}
$$

We say that a sequence of extended functions $f_{n}: X \rightarrow \mathbb{R} \cup\{+\infty\}, n \in \mathbb{N}$, converges uniformly to $f: X \rightarrow \mathbb{R} \cup\{+\infty\}$ on a set $Y \subset X$ when for all $\epsilon>0$ there exists $n_{0} \in \mathbb{N}$ such that $\left|f_{n}(x)-f(x)\right|<\epsilon$ for all $x \in Y$ and for all $n \geq n_{0}$. Recalling the above convention, this is equivalent to assert that $Y \cap \operatorname{dom} f_{n}=Y \cap \operatorname{dom} f$ for all $n \geq n_{0}$ and the restriction of $f_{n}$ to the later set converges uniformly (in the sense of finite-valued functions) to the restriction of $f_{n}$ to the same set.

Proposition 3.3. Let $f, f_{n} \in \mathcal{V}_{1}, n=1,2, \ldots$. Then $d\left(f_{n}, f\right) \rightarrow 0$ if and only if the sequence $\left\{f_{n}\right\}_{n \in \mathbb{N}}$ converges uniformly to $f$ on $B_{k}$, for all $k \in \mathbb{N}$.

Proof. It is immediate consequence of the previous lemmas.

As a consequence of Proposition 3.3 , the topology on $\mathcal{V}_{1}$ is the same for any other barrelled neighborhood of zero $\widetilde{B}$ such that there exist positive scalars $\alpha$ and $\beta$ satisfying $\alpha B \subset \widetilde{B} \subset \beta B$. If $X$ is a normed space, any barrelled neighborhood of zero $\widetilde{B}$ satisfies this condition relative to the unit ball $B$. Then the above topology is intrinsic. In the particular case that $X=\mathbb{R}^{n}$, this topology describes the uniform convergence on the compact subsets of $\mathbb{R}^{n}$ (as in [44]), and its restriction to $\Theta_{7}$ coincides with the topology of the uniform convergence introduced in [23], which is commonly used in the stability analysis in linear semi-infinite optimization. Obviously, other metrics on $\mathcal{V}_{1}$ could be considered instead of $d$. For instance,

$$
\rho(f, h):=\sup _{x \in X} \frac{|f(x)-h(x)|}{1+|f(x)-h(x)|}
$$

adopting the convention that $\frac{+\infty}{+\infty}=1$ describes the topology of the uniform convergence on the whole space $X$. The advantage of $\rho$ on $d$ is that it is always intrinsic to $X$. The serious inconvenient of $\rho$ is that the corresponding topology is too rich for developing a stability theory dealing with arbitrary perturbations of the constraint functions and the constraint set.

Now, let $C, C_{n}, n \in \mathbb{N}$, be subsets of $X$. From Proposition 3.3 (or from (3.2)), the convergence $\delta_{C_{n}} \rightarrow \delta_{C}$ as $n \rightarrow \infty$ (i.e., $\lim _{n} d\left(\delta_{C_{n}}, \delta_{C}\right) \rightarrow 0$ ) is characterized as follows:

Corollary 3.4. $\delta_{C_{n}} \rightarrow \delta_{C}$ if and only if for any $k \in \mathbb{N}$ there exists $n_{k} \in \mathbb{N}$ such that $C_{n} \cap B_{k}=C \cap B_{k}$ for all $n \geq n_{k}$.

Let $\mathcal{V}_{j}$ be the space of the constraint functions corresponding to parameter space $\Theta_{j}, j=1, \ldots, 7$, i.e., $\mathcal{V}_{1}=(\mathbb{R} \cup\{+\infty\})^{X}$ (the set of extended functions from $X$ to 
$\mathbb{R} \cup\{+\infty\})$,

$$
\begin{aligned}
& \mathcal{V}_{2}:=\left\{f \in \mathcal{V}_{1}: f \text { is lsc }\right\} \\
& \mathcal{V}_{j}:=\left\{f \in \mathcal{V}_{2}: \text { the local minima of } f \text { are global }\right\}, j=3,4, \\
& \mathcal{V}_{5}:=\left\{f \in \mathcal{V}_{3}: f \text { is convex }\right\} \\
& \mathcal{V}_{6}:=\left\{f \in \mathcal{V}_{5}: f \text { is finite-valued }\right\}, \text { and } \\
& \mathcal{V}_{7}:=X^{*}
\end{aligned}
$$

Observe that the improper function $\{+\infty\}^{X}$ (with constant value $+\infty$ ) is an accumulation point of $\mathcal{V}_{j}, j=1, \ldots, 5$, because $\{+\infty\}^{X}=\lim _{k} \delta_{\left\{x_{k}\right\}}$, where $x_{k} \in X \backslash B_{k}$ for all $k \in \mathbb{N}$. The topology of $\mathcal{V}_{7}$ describes the uniform convergence of the continuous linear functionals on $B$.

Proposition 3.5. $\left(\mathcal{V}_{j}, d\right)$ is a complete metric space for $j=2,5,6,7$.

Proof. First, we prove that $\left(\mathcal{V}_{1}, d\right)$ is complete. Let $\left\{f_{n}\right\}_{n \in \mathbb{N}}$ be a Cauchy sequence in $\left(\mathcal{V}_{1}, d\right)$, i.e., $d\left(f_{n}, f_{m}\right) \rightarrow 0$ as $n, m \rightarrow \infty$.

Let $\epsilon>0$. For any fixed $k \in \mathbb{N}$, by Lemma 3.1, there exists $\rho>0$ such that $d_{k}(f, h)<\epsilon$ for each pair $f, h \in \mathcal{V}_{1}$ satisfying $d(f, h)<\rho$.

Since $d\left(f_{n}, f_{m}\right) \rightarrow 0$ as $n, m \rightarrow \infty$, there is $n_{k}>0$ such that $d\left(f_{n}, f_{m}\right)<\rho$ and hence, $d_{k}\left(f_{n}, f_{m}\right)<\epsilon$ for all $m, n>n_{k}$. This means that

$$
\sup _{x \in B_{k}}\left|f_{n}(x)-f_{m}(x)\right|<\epsilon .
$$

By our convention, for each $x \in B_{k}$, either $f_{n}(x)=f_{m}(x)=+\infty$ or $\left\{f_{n}(x), f_{m}(x)\right\} \subset$ $\mathbb{R}$ for all $n, m>n_{k}$. In the first case, let $f(x)=+\infty$. For the second case, $\left\{f_{n}(x)\right\}_{n \in \mathbb{N}}$ is a Cauchy sequence in $\mathbb{R}$ and hence, converges to some point in $\mathbb{R}$ which we denote by $f(x)$. It is obvious that the sequence $\left\{f_{n}\right\}_{n \in \mathbb{N}}$ converges uniformly to $f$ on $B_{k}$. Since $k$ is taken arbitrarily, it follows that $f \in \mathcal{V}_{1}$ and also that $d\left(f_{n}, f\right) \rightarrow 0$ as $n \rightarrow \infty$ by Proposition 3.3.

Now we show that $\mathcal{V}_{2}$ is a closed subspace of $\mathcal{V}_{1}$. Let $\left\{f_{n}\right\}_{n \in \mathbb{N}} \subset \mathcal{V}_{2}$ be such that $d\left(f_{n}, f\right) \rightarrow 0$. We must prove that $f$ is lsc. Let $x_{0} \in X$ and $\lambda \in \mathbb{R}$ be such that $f\left(x_{0}\right)>\lambda$. Let $\left.\epsilon \in\right] 0,1\left[\right.$ such that $f\left(x_{0}\right)>\lambda+\epsilon$. Let $k, n_{0} \in \mathbb{N}$ be such that $x_{0} \in \operatorname{int} B_{k}$ and

$$
\left|f_{n}(x)-f(x)\right|<\frac{\epsilon}{2} \forall x \in B_{k} \forall n \geq n_{0} .
$$

If $f\left(x_{0}\right)=+\infty, f_{n_{0}}\left(x_{0}\right)=+\infty>\lambda+\epsilon$ by (3.3). Because $f_{n_{0}}$ is lsc, there exists $V \in \mathcal{N}\left(x_{0}\right)$ such that

$$
f_{n_{0}}(x)>\lambda+\frac{\epsilon}{2} \forall x \in V .
$$

Otherwise, from (3.3), $f_{n_{0}}\left(x_{0}\right)>f\left(x_{0}\right)-\frac{\epsilon}{2}>\lambda+\frac{\epsilon}{2}$ and we get again (3.4) for some $V \in \mathcal{N}\left(x_{0}\right)$. If $x \in V \cap \operatorname{int} B_{k}$, from (3.3) and (3.4), we get $f(x)>\lambda$. Consequently, $f$ is lsc at $x_{0}$. So $\mathcal{V}_{2}$ is closed.

The proof of the closedness of $\mathcal{V}_{5}, \mathcal{V}_{6}$, and $\mathcal{V}_{7}$ is left to the reader.

The next example shows that Proposition 3.5 is not true for $j=3,4$.

Example 3.6. Let $X=\mathbb{R}$ and $\left\{f_{n}\right\}_{n \in \mathbb{N}} \subset \mathcal{V}_{3}$ such that

$$
f_{n}(x)= \begin{cases}|x|, & \text { if } x \leq 1, \\ \frac{x+n}{n+1}, & \text { if } x \in] 1, \frac{2 n+1}{n}[, \\ x-1, & \text { if } x \geq \frac{2 n+1}{n},\end{cases}
$$


$n \in \mathbb{N}$ (the unique local minimum of $f_{n}, 0$, is global). Then $d\left(f_{n}, f\right) \rightarrow 0$, where

$$
f(x)= \begin{cases}|x|, & \text { if } x \leq 1, \\ 1, & \text { if } x \in] 1,2[, \\ x-1, & \text { if } x \geq 2,\end{cases}
$$

whose set of local minima, $\{0\} \cup] 1,2]$, only contains a global minimum, 0 , so that $f \notin \mathcal{V}_{3}$ (see Figures 1-2).

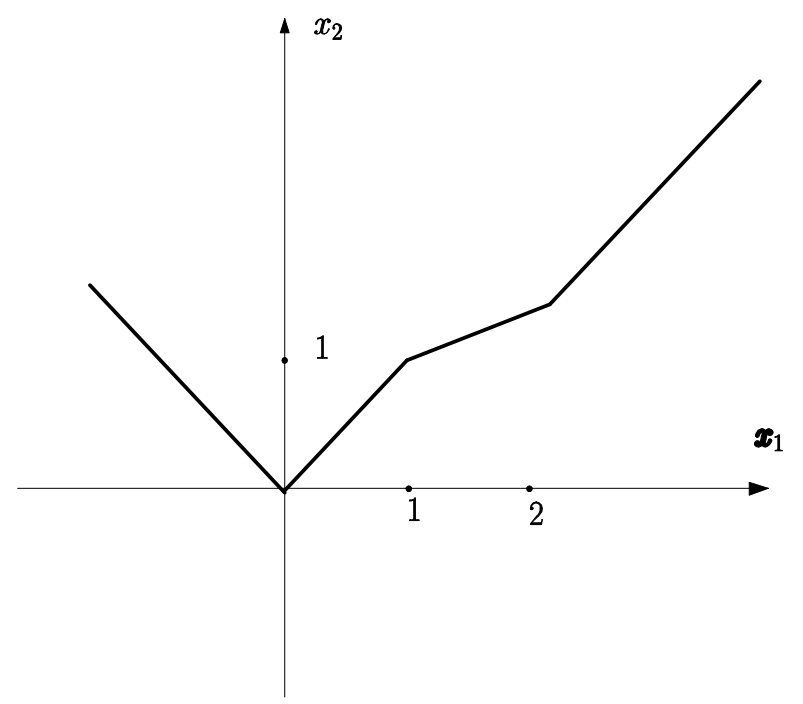

Figure 1: Graph of $f_{n}$.

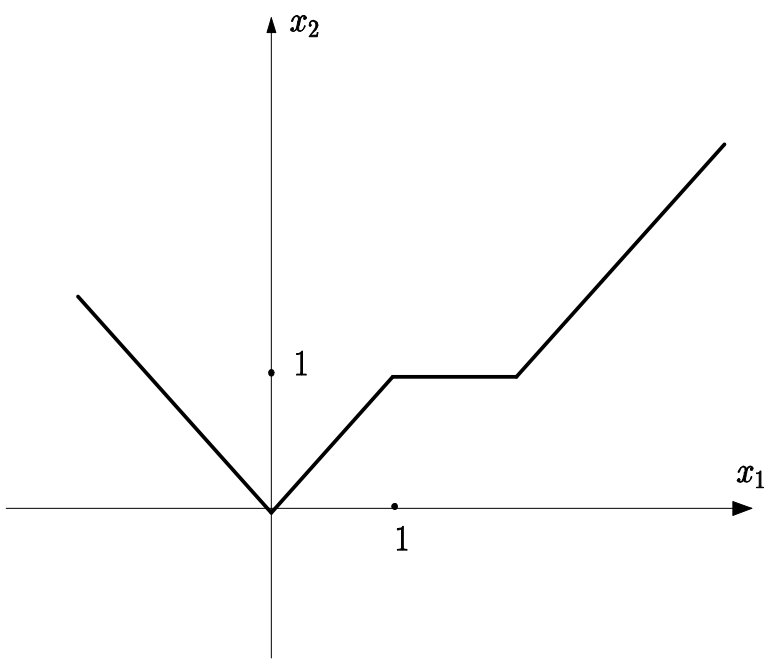

Figure 2: Graph of $f$.

Given $\sigma=\left\{f_{t}, t \in T ; C\right\}, \sigma_{1}=\left\{f_{t}^{1}, t \in T ; C_{1}\right\} \in \Theta_{j}$, we define

$$
\mathbf{d}\left(\sigma, \sigma_{1}\right):= \begin{cases}\max \left\{\sup _{t \in T} d\left(f_{t}, f_{t}^{1}\right), d\left(\delta_{C}, \delta_{C_{1}}\right)\right\}, & \text { if } T \neq \emptyset \\ d\left(\delta_{C}, \delta_{C_{1}}\right), & \text { if } T=\emptyset\end{cases}
$$


Proposition 3.7. $(\Theta, \mathbf{d})$ is a metric space for all $\Theta \subset \Theta_{1}$. Moreover, $(\Theta, \mathbf{d})$ is complete if $\Theta$ is a closed subset of $\Theta_{1}$. In particular, $\left(\Theta_{j}, \mathbf{d}\right)$ is complete, $j=2,5,6,7$.

Proof. It is sufficient to prove that $\left(\Theta_{1}, \mathbf{d}\right)$ is a complete metric space and that $\Theta_{j}$ is closed for $j=2,5,6,7$. We prove the statement assuming that $T \neq \emptyset$ (the proof is simpler if $T=\emptyset$ ).

In order to show that $\left(\Theta_{1}, \mathbf{d}\right)$ is a metric space it is sufficient to verify the triangular inequality. Let $\sigma=\left\{f_{t}, t \in T ; C\right\}, \sigma_{1}=\left\{f_{t}^{1}, t \in T ; C_{1}\right\}$ and $\sigma_{2}=\left\{f_{t}^{2}, t \in T ; C_{2}\right\}$ be systems from $\Theta_{1}$. We have

$$
\begin{aligned}
\mathbf{d}\left(\sigma, \sigma_{1}\right) & +\mathbf{d}\left(\sigma_{1}, \sigma_{2}\right)= \\
& =\max \left\{\sup _{t \in T} d\left(f_{t}, f_{t}^{1}\right), d\left(\delta_{C}, \delta_{C_{1}}\right)\right\}+\max \left\{\sup _{t \in T} d\left(f_{t}^{1}, f_{t}^{2}\right), d\left(\delta_{C_{1}}, \delta_{C_{2}}\right)\right\} \\
& \geq \max \left\{\sup _{t \in T} d\left(f_{t}, f_{t}^{1}\right)+\sup _{t \in T} d\left(f_{t}^{1}, f_{t}^{2}\right), d\left(\delta_{C}, \delta_{C_{1}}\right)+d\left(\delta_{C_{1}}, \delta_{C_{2}}\right)\right\} \\
& \geq \max \left\{\sup _{t \in T}\left\{d\left(f_{t}, f_{t}^{1}\right)+d\left(f_{t}^{1}, f_{t}^{2}\right)\right\}, d\left(\delta_{C}, \delta_{C_{1}}\right)+d\left(\delta_{C_{1}}, \delta_{C_{2}}\right)\right\} \\
& \geq \max \left\{\sup _{t \in T} d\left(f_{t}, f_{t}^{2}\right), d\left(\delta_{C}, \delta_{C_{2}}\right)\right\}=\mathbf{d}\left(\sigma, \sigma_{2}\right) .
\end{aligned}
$$

Now we prove that $\left(\Theta_{1}, \mathbf{d}\right)$ is complete. Let $\left\{\sigma_{n}\right\}_{n \in \mathbb{N}}$ be a Cauchy sequence in $\left(\Theta_{1}, \mathbf{d}\right)$.

We first prove its convergence for the case where $C=X$. Suppose that $\sigma_{n}=$ $\left\{f_{t}^{n}, t \in T ; X\right\}$ for all $n \in \mathbb{N}$. Let $\left.\epsilon \in\right] 0,1[$ be fixed. We must show that there is a system $\sigma \in \Theta_{1}$ such that $\mathbf{d}\left(\sigma_{n}, \sigma\right) \rightarrow 0$ as $n$ tends to infinity. For any $k \in \mathbb{N}$, by Lemma 3.1, there is $\rho_{k}>0$ such that

$$
d(f, h)<\rho_{k} \Rightarrow d_{k}(f, h)<\epsilon .
$$

As $\left\{\sigma_{n}\right\}_{n \in \mathbb{N}}$ is a Cauchy sequence, there exists $n_{0}>0$ such that for any $m, n \geq n_{0}$, one has

$$
\mathbf{d}\left(\sigma_{n}, \sigma_{m}\right)=\sup _{t \in T} d\left(f_{t}^{n}, f_{t}^{m}\right)<\rho_{k}
$$

which gives

$$
d\left(f_{t}^{n}, f_{t}^{m}\right)<\rho_{k}, \quad \forall t \in T, \forall m, n \geq n_{0} .
$$

It follows from (3.6) that

$$
d_{k}\left(f_{t}^{n}, f_{t}^{m}\right)=\sup _{x \in B_{k}}\left|f_{t}^{n}(x)-f_{t}^{m}(x)\right|<\epsilon, \quad \forall t \in T, \forall m, n \geq n_{0} .
$$

By an argument as in the proof of Proposition 3.5, for each $t \in T$, there exists a function $f_{t} \in \mathcal{V}_{1}$ such that $d\left(f_{t}^{n}, f_{t}\right) \rightarrow 0$ as $n \rightarrow \infty$. Let $\sigma:=\left\{f_{t}, t \in T ; X\right\}$.

We now prove that $\mathbf{d}\left(\sigma_{n}, \sigma\right) \rightarrow 0$ as $n \rightarrow \infty$.

With $\epsilon>0$ fixed, by Lemma 3.2, there exist $k_{0}$ and $\rho_{0}>0$ such that for any $f, h \in \mathcal{V}_{1}$,

$$
d_{k_{0}}(f, h)<\rho_{0} \Rightarrow d(f, h)<\epsilon .
$$

Since $\mathbf{d}\left(\sigma_{n}, \sigma_{m}\right) \rightarrow 0$, there exists $n_{1}>0$ such that for all $n, m \geq n_{1}$,

$$
\mathbf{d}\left(\sigma_{n}, \sigma_{m}\right)=\sup _{t \in T} d\left(f_{t}^{n}, f_{t}^{m}\right)=\sup _{t \in T} \sum_{i=1}^{\infty} 2^{-i} d_{i}\left(f_{t}^{n}, f_{t}^{m}\right)<\rho_{0} 2^{-k_{0}} .
$$


This yields

$$
d_{k_{0}}\left(f_{t}^{n}, f_{t}^{m}\right)<\rho_{0}, \forall t \in T
$$

which, in turn, implies that (letting $m \rightarrow \infty$ )

$$
d_{k_{0}}\left(f_{t}^{n}, f_{t}\right) \leq \rho_{0}, \quad \forall t \in T, \forall n \geq n_{1} .
$$

By (3.9), the last inequality yields

$$
\sup _{t \in T} d\left(f_{t}^{n}, f_{t}\right) \leq \epsilon, \forall n \geq n_{1} .
$$

Therefore, $\mathbf{d}\left(\sigma_{n}, \sigma\right) \rightarrow 0$ as $n$ tends to $\infty$.

We now turn to the case where $\sigma_{n}=\left\{f_{t}^{n}, t \in T ; C_{n}\right\}$ for all $n \in \mathbb{N}$. Since $\left\{\sigma_{n}\right\}_{n \in \mathbb{N}}$ is a Cauchy sequence, $d\left(\delta_{C_{n}}, \delta_{C_{m}}\right) \rightarrow 0$ as $n, m$ tends to infinity. By the completeness of $\left(\mathcal{V}_{1}, d\right)$, there exists $h \in \mathcal{V}_{1}$ such that $d\left(\delta_{C_{n}}, h\right) \rightarrow 0$ as $n \rightarrow \infty$. Since $\delta_{C_{n}} \rightarrow h$ pointwise, $h(x) \in\{0,+\infty\}$ for all $x \in X$. Then $h=\delta_{C}$, where $C:=\operatorname{dom} h \neq \emptyset$. Then $d\left(\delta_{C_{n}}, \delta_{C}\right) \rightarrow 0$ as $n \rightarrow \infty$.

Let $\sigma:=\left\{f_{t}, t \in T ; C\right\}$. Combining the two parts of the proof, we conclude that $\mathbf{d}\left(\sigma_{n}, \sigma\right) \rightarrow 0$ as $n \rightarrow \infty$. Consequently, $\left(\Theta_{1}, \mathbf{d}\right)$ is complete.

The closedness of $\Theta_{j}, j=2,5,6,7$, follows from Proposition 3.5.

Consider the sequence $\left\{\sigma_{n}\right\}_{n \in \mathbb{N}}$ such that $\sigma_{n}=\left\{f_{n} ; \mathbb{R}\right\} \subset \Theta_{3}$, where $f_{n}$ is the function defined in Example 3.6. It is easy to see that $\mathbf{d}\left(\sigma_{n}, \sigma\right) \rightarrow 0$, where $\sigma=$ $\{f ; \mathbb{R}\} \notin \Theta_{3}$. Thus $\Theta_{3}$ is not closed.

In the rest of the paper, for the sake of simplicity, we will write $d\left(\sigma, \sigma_{1}\right)$ instead of $\mathbf{d}\left(\sigma, \sigma_{1}\right)$ whenever there is no ambiguity.

The next example emphasizes that the properties of the feasible set mapping at the nominal system $\sigma$ are not determined by its feasible set $F$ (recall that $\Theta$ depends on the index set, and so on the particular form of $\sigma$ ).

ExAmple 3.8. Let $C \subset X$ be a nonempty closed convex set. We analyze the lsc property of the feasible set mapping at three different representations of $C$, with $T$ empty, singleton, and $T$ infinite, respectively.

(a) Let $\sigma=\{x \in C\} \in \Theta_{5}$. The elements of $\Theta_{5}$ can be expressed here as $\sigma_{1}=\left\{x \in C_{1}\right\}$ with $C_{1} \subset X$ nonempty, closed and convex. Let $W$ be an open set in $X$ such that $W \cap C \neq \emptyset$. Take $\bar{x} \in W \cap C$. Let $\bar{k} \in \mathbb{N}$ be such that $\bar{x} \in B_{\bar{k}}$. If $\bar{x} \notin C_{1}$ then $\bar{x} \in\left(B_{\bar{k}} \cap C\right) \backslash\left(B_{\bar{k}} \cap C_{1}\right)$ so that $k_{1} \leq \bar{k}$ ( $k_{1}$ was defined in (3.1)). Thus, $\bar{k}<k_{1}$ implies $\bar{x} \in C_{1}$. Consequently, $d\left(\sigma, \sigma_{1}\right)=d\left(\delta_{C_{1}}, \delta_{C}\right)<2^{1-\bar{k}}$ implies that $\bar{k}<k_{1}$ (according to (3.1)), so that $\bar{x} \in C_{1}$ and $\mathcal{F}_{5}\left(\sigma_{1}\right) \cap W=C_{1} \cap W \neq \emptyset$. Then $\mathcal{F}_{5}$ is lsc at $\sigma$.

(b) $\mathcal{F}_{5}$ is not lsc at $\sigma=\left\{\delta_{C}(x) \leq 0 ; x \in X\right\} \in \Theta_{5}$ because $\sigma_{1}:=\left\{\delta_{C}(x)+\epsilon \leq 0 ; x \in\right.$ $X\} \notin \operatorname{dom} \mathcal{F}_{5}$ for $\epsilon=d\left(\sigma, \sigma_{1}\right)>0$ arbitrarily small.

(c) Let $\sigma=\left\{v(x) \leq \alpha,(v, \alpha) \in\right.$ epi $\left.\delta_{C}^{*}+(0,1) ; x \in X\right\} \in \Theta_{7}$, obtained from (2.5). It is easy to show that any solution of $\sigma$ is an SS-point of $\sigma$. In $\$ 5$ we prove that the strong Slater condition characterizes the lsc property of $\mathcal{F}_{7}$ at $\sigma$. Observe that the elimination of " $+(0,1)$ " from the index set of $\sigma$ provides another linear representation of $C$ (from (2.4)) such that $\mathcal{F}_{7}$ cannot be lsc at that system because arbitrarily small perturbations of $(0,0) \in \operatorname{epi} \delta_{C}^{*}$ of the type $(0,-\epsilon)$ provide inconsistent systems. 
4. Closedness. Being $\mathcal{F}(\sigma)$ a closed subset of $X$ for all $\sigma \in \Theta$ is a necessary condition for the closedness of $\mathcal{F}: \Theta \rightrightarrows X$. Thus $\mathcal{F}_{1}$ is not closed (consider $\sigma=$ $\left\{f_{t}, t \in T ; C\right\}$ such that $f_{t}=0$ for all $t \in T$ and $C$ is nonclosed). The feasible set mapping $\mathcal{F}$ satisfies this necessary condition when $\Theta \subset \Theta_{2}$, which turns out to be also sufficient according to the next result.

ThEOREM 4.1. $\mathcal{F}$ is closed for any $\Theta \subset \Theta_{2}$.

Proof. We assume that $T \neq \emptyset$. It is enough to prove that $\mathcal{F}_{2}$ is closed because $\mathcal{F}$ is the restriction of $\mathcal{F}_{2}$ to $\Theta \subset \Theta_{2}$.

Consider $\sigma=\left\{f_{t}, t \in T ; C\right\} \in \Theta_{2}$. Let $\left\{\sigma_{\delta}\right\}_{\delta \in \Delta} \subset \Theta_{2}$, where $\sigma_{\delta}=\left\{f_{t}^{\delta}, t \in\right.$ $\left.T ; C_{\delta}\right\}, \delta \in \Delta$, and $\left\{x_{\delta}\right\}_{\delta \in \Delta} \subset X$ be nets satisfying

$$
\lim _{\delta} \sigma_{\delta}=\sigma, \lim _{\delta} x_{\delta}=\widetilde{x} \text {, and } x_{\delta} \in \mathcal{F}_{2}\left(\sigma_{\delta}\right) \text { for all } \delta \in \Delta .
$$

We will show that $\widetilde{x} \in \mathcal{F}_{2}(\sigma)$. To this aim, we observe firstly that for any fixed $\bar{t} \in T$, we have

$$
f_{\bar{t}}(\widetilde{x}) \leq \liminf _{\delta} f_{\bar{t}}\left(x_{\delta}\right)
$$

by the lower semicontinuity of $f_{\bar{t}}$. On the other hand, since

$$
\lim _{\delta} d\left(\sigma, \sigma_{\delta}\right)=\lim _{\delta} \max \left\{\sup _{t} d\left(f_{t}, f_{t}^{\delta}\right), d\left(\delta_{C}, \delta_{C_{\delta}}\right)\right\}=0
$$

one gets $\lim _{\delta} d\left(f_{\bar{t}}, f_{\bar{t}}^{\delta}\right)=0$, which, together with Lemma 3.1, gives

$$
\lim _{\delta} d_{k}\left(f_{\bar{t}}, f_{\bar{t}}^{\delta}\right)=0, \quad \forall k \in \mathbb{N} .
$$

Since $\left\{x_{\delta}\right\}_{\delta \in \Delta}$ is a convergent net, without loss of generality, assume that $\left\{x_{\delta}\right\}_{\delta \in \Delta} \subset$ $\operatorname{int} B_{k}$ for $k \in \mathbb{N}$ large enough. By definition of $d_{k}$, for each $\delta \in \Delta$,

$$
\left|f_{\bar{t}}\left(x_{\delta}\right)-f_{\bar{t}}^{\delta}\left(x_{\delta}\right)\right| \leq d_{k}\left(f_{\bar{t}}, f_{\bar{t}}^{\delta}\right)
$$

and hence,

$$
f_{\bar{t}}\left(x_{\delta}\right) \leq f_{\bar{t}}^{\delta}\left(x_{\delta}\right)+d_{k}\left(f_{\bar{t}}, f_{\bar{t}}^{\delta}\right) \leq d_{k}\left(f_{\bar{t}}, f_{\bar{t}}^{\delta}\right), \forall \delta \in \Delta
$$

as $f_{\bar{t}}\left(x_{\delta}\right) \leq 0$ by the fact that $x_{\delta} \in \mathcal{F}_{2}\left(\sigma_{\delta}\right)$. Combining this, (4.3), and (4.2) we get $f_{\bar{t}}(\widetilde{x}) \leq 0$.

By the same argument as above, recalling that $C$ is closed, we can prove that $\delta_{C}(\widetilde{x}) \leq 0$ or $\widetilde{x} \in C$. Consequently, $\widetilde{x} \in \mathcal{F}_{2}(\sigma)$.

Obviously, the feasible set mapping $\mathcal{F}$ is still closed for $\Theta \subset \Theta_{2}$ when the only admissible perturbations involve the RHS function. The next result is a consequence of Theorem 4.1 and the axiom of choice.

Proposition 4.2. Let $\Theta \subset \Theta_{2}$. If $\left\{\sigma_{\delta}\right\}_{\delta \in \Delta} \subset \operatorname{dom} \mathcal{F}$ and $\lim _{\delta} \sigma_{\delta}=\sigma$, then $\operatorname{Ls}_{\delta} \mathcal{F}\left(\sigma_{\delta}\right) \subset \mathcal{F}(\sigma)$

Proof. Take a fixed $x_{0} \in \operatorname{Ls}_{\delta} \mathcal{F}\left(\sigma_{\delta}\right)$, and consider the set

$$
\Lambda:=\left\{(\delta, U) \in \Delta \times \mathcal{N}\left(x_{0}\right): \mathcal{F}\left(\sigma_{\delta}\right) \cap U \neq \emptyset\right\} .
$$

Observe that $\Lambda$ is a directed set via the product order

$$
(\delta, U) \leq\left(\delta^{\prime}, U^{\prime}\right) \Leftrightarrow \delta \preceq \delta^{\prime} \text { and } U \supset U^{\prime} .
$$


Therefore, by picking for each $(\delta, U) \in \Lambda$ a point $x_{(\delta, U)} \in \mathcal{F}\left(\sigma_{\delta}\right) \cap U$, we build a net $\left\{x_{(\delta, U)}\right\}_{(\delta, U) \in \Lambda}$ in $X$. It is easy to see that this net converges to the point $x_{0}$.

Through $(\Lambda, \leq)$ we shall generate a subnet of $\left\{\sigma_{\delta}\right\}_{\delta \in \Delta}$. In fact if we consider the function $h: \Lambda \rightarrow \Delta$ defined by $h(\delta, U)=\delta$, the following conditions are satisfied:

(a) $(\delta, U) \leq\left(\delta^{\prime}, U^{\prime}\right) \Rightarrow h(\delta, U) \preceq h\left(\delta^{\prime}, U^{\prime}\right)$.

(b) The set $h(\Lambda)$ is cofinal in $(\Delta, \preceq)$. To see this, take a fixed $\delta \in \Delta$ and any $U \in \mathcal{N}\left(x_{0}\right)$. Then, there will exist $\delta^{\prime} \in \Delta$ such that $\delta \preceq \delta^{\prime}$ and $\mathcal{F}\left(\sigma_{\delta^{\prime}}\right) \cap U \neq \emptyset$. Therefore, $\left(\delta^{\prime}, U\right) \in \Lambda$ and $\delta \preceq h\left(\delta^{\prime}, U\right)$.

As a consequence of (a) and (b), $\left\{\sigma_{h(\delta, U)}\right\}_{(\delta, U) \in \Lambda}$ is a subnet of $\left\{\sigma_{\delta}\right\}_{\delta \in \Delta}$ and, so, $\lim _{(\delta, U)} \sigma_{h(\delta, U)}=\sigma$. Then, the closedness of $\mathcal{F}$ and $x_{(\delta, U)} \in \mathcal{F}\left(\sigma_{\delta}\right)=\mathcal{F}\left(\sigma_{h(\delta, U)}\right)$ together entail $x_{0} \in \mathcal{F}(\sigma)$.

ExAmple 4.3. Let $\sigma=\{f ; \mathbb{R}\}$ be such that

$$
f(x)= \begin{cases}x^{2}-2, & \text { if } x \in D \\ 1, & \text { if } x= \pm 1 \\ +\infty, & \text { otherwise }\end{cases}
$$

where $D=]-1,1\left[\right.$ (see Figure 3). It is easy to show that $\sigma \in \Theta_{1} \backslash \Theta_{2}, \mathcal{F}_{1}$ is not closed at $\sigma$ (because $\mathcal{F}_{1}(\sigma)=D$ is nonclosed) and, taking a constant net $\sigma_{\delta}=\sigma$ for all $\delta \in \Delta$, we get $\operatorname{Ls}_{\delta} \mathcal{F}_{1}\left(\sigma_{\delta}\right)=\operatorname{cl} D \nsubseteq \mathcal{F}_{1}(\sigma)=D$. Thus the results in this section fail for $\mathcal{F}_{1}$.

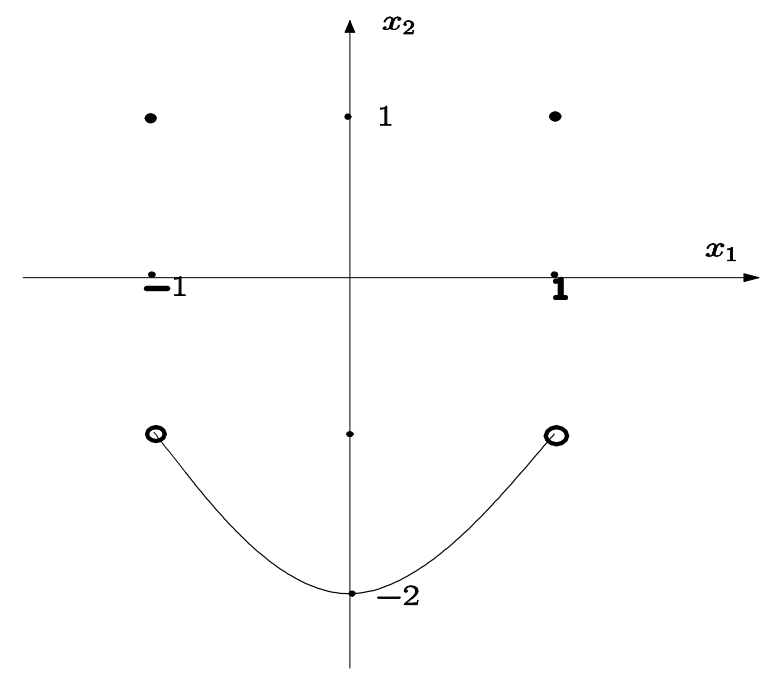

Figure 3: Graph of $f$

5. Lower semicontinuity. The main result of the paper provides different characterizations of the lower semicontinuity of the feasible set mapping.

Theorem 5.1. $\mathcal{F}$ is lsc if $T=\emptyset$ and $\Theta \subset \Theta_{1}$. Otherwise, consider the following statements associated with $\sigma=\left\{f_{t}, t \in T ; C\right\} \in \operatorname{dom} \mathcal{F}$ :

(i) $\mathcal{F}$ is lsc at $\sigma$;

(ii) $\sigma \in \operatorname{int} \operatorname{dom} \mathcal{F}$;

(iii) $\sigma$ is Tuy regular;

(iv) $\sigma$ satisfies the strong Slater condition;

(v) $F=\operatorname{cl} F_{S S}$, where $F_{S S}$ is the set of all strong Slater points of $\sigma$; 
(vi) For every net $\left\{\sigma_{\delta}\right\}_{\delta \in \Delta}$ converging to $\sigma$, the net of sets $\left\{\mathcal{F}\left(\sigma_{\delta}\right)\right\}_{\delta \in \Delta}$ converges to $\mathcal{F}(\sigma)$ in the sense of Kuratowski-Painlevé;

(vii) $0 \notin \operatorname{clconv}\left\{\left(\bigcup_{t \in T} \operatorname{epi} f_{t}^{*}\right)+\operatorname{epi} \delta_{C}^{*}\right\}$; and

(viii) $0 \notin \operatorname{cl} \operatorname{conv}\left\{\left(\bigcup_{t \in T} \operatorname{epi} f_{t}^{*}\right) \cup\left[\operatorname{epi} \delta_{C}^{*}+(0,1)\right]\right\}$.

Then, (i) $\Rightarrow$ (ii) $\Rightarrow$ (iii) $\Rightarrow$ (iv) if $\sigma \in \Theta_{1}$, (i)-(vi) are equivalent to each other if $\sigma \in$ $\Theta_{3} \cup \Theta_{5}$, and (i)-(viii) are equivalent to each other if $\sigma \in \Theta_{5}$.

Proof. Let $T=\emptyset$ and $\Theta \subset \Theta_{1}$. $\mathcal{F}$ is trivially lsc at any $\sigma \in \Theta \backslash(\operatorname{dom} \mathcal{F})$, and also at any $\sigma \in \operatorname{dom} \mathcal{F}$ by the argument of Example 3.8 (a). Thus we can assume that $T \neq \emptyset$ and $\sigma=\left\{f_{t}, t \in T ; C\right\} \in \operatorname{dom} \mathcal{F}$. We shall prove that $(i) \Rightarrow(i i) \Rightarrow(i i i) \Rightarrow(i v)$ if $\sigma \in \Theta_{1}$, that $(i v) \Rightarrow(i),(i) \Rightarrow(v) \Rightarrow(i v)$, and $(i) \Rightarrow(v i) \Rightarrow(i i)$ if $\sigma \in \Theta_{3} \cup \Theta_{5}$, and, finally, that $(v i i) \Leftrightarrow(i v) \Leftrightarrow(v i i i)$ if $\sigma \in \Theta_{5}$.

(i) $\Rightarrow$ (ii) Let $\sigma \in \Theta \subset \Theta_{1}$. Suppose that $\mathcal{F}$ is lsc at $\sigma$. Then for any open subset $W \subset X$ with $W \cap \mathcal{F}(\sigma) \neq \emptyset$, there exists $\epsilon>0$ such that for any $\sigma_{1} \in \Theta, d\left(\sigma, \sigma_{1}\right)<\epsilon$ implies $\mathcal{F}\left(\sigma_{1}\right) \cap W \neq \emptyset$, which proves that $\sigma \in \operatorname{int} \operatorname{dom} \mathcal{F}$.

(ii) $\Rightarrow$ (iii) Let $\sigma \in \Theta \subset \Theta_{1}$. Suppose that $\sigma \in \operatorname{int} \operatorname{dom} \mathcal{F}$. Let $\epsilon>0$ be a number such that

$$
d\left(\sigma, \sigma_{1}\right) \leq \epsilon \quad \sigma_{1} \in \operatorname{dom} \mathcal{F} .
$$

By Lemma 3.2, there exist $k \in \mathbb{N}$ and $\rho>0$ such that $d(f, h)<\epsilon$ for each pair of functions $f, h \in \mathcal{V}_{j}$ satisfying $d_{k}(f, h)<\rho$. Without loss of generality, we can assume that $\rho<\epsilon$. Let $w \in \mathbb{R}^{T}$ and $C_{1} \subset X$ such that $\sigma_{1}:=\left\{f_{t}(x)-w_{t} \leq 0, t \in T ; x \in\right.$ $\left.C_{1}\right\} \in \Theta$ and

$$
\max \left\{\sup _{t \in T}\left|w_{t}\right|, d\left(\delta_{C}, \delta_{C_{1}}\right)\right\}<\rho<\epsilon
$$

Then for any $t \in T, d_{k}\left(f_{t}^{1}, f_{t}\right)<\rho$, which implies $d\left(f_{t}^{1}, f_{t}\right)<\epsilon$. In turn, this and (5.2) imply $d\left(\sigma, \sigma_{1}\right)<\epsilon$ and hence, $\sigma_{1} \in \operatorname{dom} \mathcal{F}$ by (5.1). Thus, $\sigma$ is Tuy regular.

(iii) $\Rightarrow\left(\right.$ iv) Let $\sigma \in \Theta \subset \Theta_{1}$. Suppose now that $\sigma$ is Tuy regular. Then, for some $\epsilon>0$, the system $\sigma_{1}=\left\{f_{t}-w_{t}, t \in T ; C_{1}\right\} \in \Theta$ is consistent whenever

$$
\max \left\{\sup _{t \in T}\left|w_{t}\right| ; d\left(\delta_{C}, \delta_{C_{1}}\right)\right\}<\epsilon .
$$

Let $w_{t}=-\frac{\epsilon}{2}$ for all $t \in T$ and $C_{1}=C$. Since $\sigma_{1} \in \Theta$ (because $\Theta$ is closed w.r.t. constant perturbations of the RHS function) and (5.3) holds, $\sigma_{1}$ is consistent. It is obvious that any $\tilde{x} \in \mathcal{F}\left(\sigma_{1}\right)$ is a SS-point of $\sigma$.

(iv) $\Rightarrow$ (i) Let $\sigma \in \Theta_{3} \cup \Theta_{5}$. Let $W \subset X$ be an open set such that $W \cap \mathcal{F}(\sigma) \neq \emptyset$. Let $\bar{x} \in W \cap \mathcal{F}(\sigma)$, and $\hat{x} \in C$ and $\rho>0$ such that $f_{t}(\hat{x}) \leq-\rho$ for all $t \in T$.

First we assume $\sigma \in \Theta_{3}$. Since $\mathcal{F}(\sigma) \subset \operatorname{int} C$, we can assume $W \subset \operatorname{int} C$ without loss of generality.

If $g(\bar{x})=0$, then $\bar{x}$ is not a global optimizer of $g$ because $g(\hat{x}) \leq-\rho<0$. Since $g \in \mathcal{V}_{3}, \bar{x}$ cannot be local minimum of $g$ so that there exists some $\widetilde{x} \in W$ such that 
$g(\widetilde{x})<0$. Let $\eta:=-g(\widetilde{x})>0$ and $\widetilde{k} \in \mathbb{N}$ be such that $\widetilde{x} \in B_{\widetilde{k}}$. Let $\epsilon>0$ such that $d\left(\sigma_{1}, \sigma\right)<\epsilon$ implies that $\left|f_{t}^{1}(\widetilde{x})-f_{t}(\widetilde{x})\right|<\frac{\eta}{2}$ for all $t \in T$. Then

$$
f_{t}^{1}(\widetilde{x})<f_{t}(\widetilde{x})+\frac{\eta}{2} \leq g(\widetilde{x})+\frac{\eta}{2}=-\frac{\eta}{2} \text { for all } t \in T .
$$

On the other hand, since $\widetilde{x} \in W \subset C$, by the argument of Example 3.8 (a), $d\left(\sigma_{1}, \sigma\right)<2^{1-\widetilde{k}}$ implies that $\widetilde{x} \in C_{1}$. Hence, $\widetilde{x} \in W \cap \mathcal{F}\left(\sigma_{1}\right)$ whenever $\sigma_{1} \in \Theta_{3}$ satisfies $d\left(\sigma_{1}, \sigma\right)<\min \left\{\epsilon, 2^{1-\widetilde{k}}\right\}$.

Now we assume $\sigma \in \Theta_{5}$. For $\left.\left.\lambda \in\right] 0,1\right]$, we consider $x(\lambda)=(1-\lambda) \bar{x}+\lambda \hat{x} \in C$. This is a SS-point of $\sigma$ because, for each $t \in T$, we have

$$
f_{t}(x(\lambda)) \leq(1-\lambda) f_{t}(\bar{x})+\lambda f_{t}(\hat{x}) \leq-\lambda \rho .
$$

Let $k \in \mathbb{N}$ be such that $\hat{x}, \bar{x} \in B_{k}$, so that $x(\lambda) \in B_{k}$ for all $\left.\left.\lambda \in\right] 0,1\right]$. Recalling the reasoning in Example 3.8 (a), for every closed convex set $C_{1}$, it holds

$$
\left.\left.d\left(\delta_{C_{1}}, \delta_{C}\right)<2^{1-k} \Rightarrow x(\lambda) \in C_{1} \forall \lambda \in\right] 0,1\right] .
$$

On the other hand, by Lemma 3.1, there exists $\rho>0$ such that, for every $t \in T$,

$$
d\left(f_{t}^{1}, f\right)<\rho \Rightarrow d_{k}\left(f_{t}^{1}, f_{t}\right)<1 .
$$

So, if $\sigma_{1}=\left\{f_{t}^{1}, t \in T ; C_{1}\right\} \in \Theta$ satisfies $d\left(\sigma_{1}, \sigma\right)<\rho$, then

$$
\begin{aligned}
\left|f_{t}^{1}(x(\lambda))-f_{t}(x(\lambda))\right| & \leq d_{k}\left(f_{t}^{1}, f_{t}\right)=\min \left\{1, d_{k}\left(f_{t}^{1}, f_{t}\right)\right\} \\
& \leq 2^{k} d\left(f_{t}^{1}, f_{t}\right) \leq 2^{k} d\left(\sigma_{1}, \sigma\right) .
\end{aligned}
$$

Let $\lambda \in] 0,1]$. According to (5.4), (5.5), (5.6), and (5.7), if

$$
d\left(\sigma_{1}, \sigma\right)<\min \left\{2^{1-k}, \rho, 2^{-k} \lambda \rho\right\}
$$

(positive number depending on $\lambda$ ), we have $x(\lambda) \in C_{1}$ and

$$
f_{t}^{1}(x(\lambda)) \leq f_{t}(x(\lambda))+2^{k} d\left(\sigma_{1}, \sigma\right)<0, \forall t \in T,
$$

i.e., $x(\lambda) \in \mathcal{F}\left(\sigma_{1}\right)$ for $\sigma_{1}$ close enough to $\sigma$.

Since $\lim _{\lambda \rightarrow 0} x(\lambda)=\bar{x} \in W, x(\lambda) \in W$ for $\lambda>0$ small enough, so that $W \cap$ $\mathcal{F}\left(\sigma_{1}\right) \neq \emptyset$ for $\sigma_{1}$ close enough to $\sigma$.

$(i) \Rightarrow(v)$ Let $\sigma \in \Theta \subset \Theta_{2}$. Since $F_{S S} \subset \mathcal{F}(\sigma)$ and $\mathcal{F}(\sigma)$ is closed, we get $\operatorname{cl}\left(F_{S S}\right) \subset \mathcal{F}(\sigma)$. We now suppose that $\mathcal{F}(\sigma) \backslash \operatorname{cl}\left(F_{S S}\right) \neq \emptyset$. Then for any point $x_{1}$ in this set, there exists an open set $W$ with $x_{1} \in W$ and $W \cap \operatorname{cl}\left(F_{S S}\right)=\emptyset$. Since $\mathcal{F}$ is lsc at $\sigma$, there is $\epsilon>0$ such that $\mathcal{F}\left(\sigma_{1}\right) \cap W \neq \emptyset$ whenever $d\left(\sigma, \sigma_{1}\right) \leq \epsilon$. Take $\sigma_{1}:=\left\{f_{t}+\epsilon, t \in T ; C\right\} \in \Theta$. Then it is clear that $d\left(\sigma, \sigma_{1}\right)=\epsilon$ and $\mathcal{F}\left(\sigma_{1}\right) \subset F_{S S}$. By the lsc property of $\mathcal{F}, W \cap \mathcal{F}\left(\sigma_{1}\right) \neq \emptyset$, which contradicts the fact that $W \cap \operatorname{cl}\left(F_{S S}\right)=\emptyset$. Therefore, $\operatorname{cl}\left(F_{S S}\right)=\mathcal{F}(\sigma)$.

$(v) \Rightarrow(i v)$ It is obvious since $\operatorname{cl}\left(F_{S S}\right)=\mathcal{F}(\sigma) \neq \emptyset$.

$(i) \Rightarrow(v i)$ Let $\sigma \in \Theta \subset \Theta_{2}$. The inclusion $\operatorname{Li}_{\delta} \mathcal{F}\left(\sigma_{\delta}\right) \subset \mathcal{F}(\sigma)$ is a consequence of the lower semicontinuity of $\mathcal{F}$ at $\sigma$ (by the same argument as in [47, Section 5B]). In order to prove the reverse inclusion, consider an arbitrary $x_{0} \in \mathcal{F}(\sigma)$. Since $\mathcal{F}$ 
is lsc at $\sigma$, for each $U \in \mathcal{N}\left(x_{0}\right)$ there will exist a neighborhood $V$ of $\sigma$ such that $U \cap \mathcal{F}\left(\sigma^{\prime}\right) \neq \emptyset$ for each $\sigma^{\prime} \in V$. Assume that $\delta_{0} \in \Delta$ is such that $\delta \in \Delta$ and $\delta_{0} \preceq \delta$ entail $\sigma_{\delta} \in V$ and, hence, $U \cap \mathcal{F}\left(\sigma_{\delta}\right) \neq \emptyset$. Consequently $x_{0} \in \operatorname{Li}_{\delta} \mathcal{F}\left(\sigma_{\delta}\right)$, and we have actually proved that $\mathcal{F}(\sigma) \subset \mathrm{Li}_{\delta} \mathcal{F}\left(\sigma_{\delta}\right)$. Since Proposition 4.2 has already established that $\operatorname{Ls}_{\delta} \mathcal{F}\left(\sigma_{\delta}\right) \subset \mathcal{F}(\sigma)$, the general inclusion $\operatorname{Li}_{\delta} \mathcal{F}\left(\sigma_{\delta}\right) \subset \operatorname{Ls}_{\delta} \mathcal{F}\left(\sigma_{\delta}\right)$ gives rise to the aimed equality $\operatorname{Li}_{\delta} \mathcal{F}\left(\sigma_{\delta}\right)=\operatorname{Ls}_{\delta} \mathcal{F}\left(\sigma_{\delta}\right)$.

$(v i) \Rightarrow(i i)$ Let $\sigma \in \Theta \subset \Theta_{1}$. Reasoning by contradiction, if (ii) does not hold, i.e., $\sigma \notin$ int $\operatorname{dom} \mathcal{F}$, we can find a sequence $\left\{\sigma_{k}\right\}_{k \in \mathbb{N}}$ converging to $\sigma$ such that $\mathcal{F}\left(\sigma_{k}\right)=\emptyset$, $k=1,2, \ldots$, and so $\operatorname{Li}_{k} \mathcal{F}\left(\sigma_{k}\right)=\operatorname{Ls}_{k} \mathcal{F}\left(\sigma_{k}\right)=\emptyset \neq \mathcal{F}(\sigma)$, which contradicts $(v i)$.

$(i v) \Leftrightarrow(v i i)$ We have $\operatorname{dom}\left(\sup _{t \in T} f_{t}\right) \cap C \neq \emptyset$ because $\sigma \in \operatorname{dom} \mathcal{F}$. By $(2.1)$ and [7, Corollary 6(b)] one has

$$
\begin{aligned}
& \operatorname{cl} \operatorname{conv}\left(\bigcup_{t \in T} \text { epi } f_{t}^{*}+\operatorname{epi} \delta_{C}^{*}\right)=\operatorname{cl}\left(\operatorname{cl} \operatorname{conv}\left(\bigcup_{t \in T} \text { epi } f_{t}^{*}\right)+\operatorname{epi} \delta_{C}^{*}\right)
\end{aligned}
$$

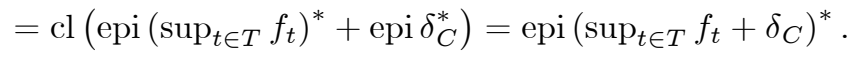

Thus (vii) holds if and only if $0 \notin \operatorname{epi}\left(\sup _{t \in T} f_{t}+\delta_{C}\right)^{*}$, i.e., $\left(\sup _{t \in T} f_{t}+\delta_{C}\right)^{*}(0)>$ 0 , if and only if there exist $\rho>0$ such that $\left(\sup _{t \in T} f_{t}+\delta_{C}\right)^{*}(0)>\rho$ if and only if there exist $\rho>0$ and $\bar{x} \in C$ such that $f_{t}(\bar{x})<-\rho$ for all $t \in T$ if and only if (iv) is true.

$($ iv $) \Leftrightarrow($ viii $)$ We have $\operatorname{dom}\left(\sup _{t \in T}\left\{f_{t}, \delta_{C}-1\right\}\right) \neq \emptyset$. Applying again (2.1) it follows that

$$
\begin{gathered}
\operatorname{cl} \operatorname{conv}\left(\bigcup_{t \in T} \text { epi } f_{t}^{*} \cup\left[\operatorname{epi} \delta_{C}^{*}+(0,1)\right]\right)=\operatorname{cl} \operatorname{conv}\left(\bigcup_{t \in T} \text { epi } f_{t}^{*} \cup \operatorname{epi}\left(\delta_{C}-1\right)^{*}\right) \\
=\operatorname{epi}\left(\sup _{t \in T}\left\{f_{t}, \delta_{C}-1\right\}\right)^{*} .
\end{gathered}
$$

Thus (viii) holds if and only if $\left(\sup _{t \in T}\left\{f_{t}, \delta_{C}-1\right\}\right)^{*}(0)>0$ if and only if there exist $\rho>0, \rho<1$, such that $\left.\sup _{t \in T}\left\{f_{t}, \delta_{C}-1\right\}\right)^{*}(0)>\rho$ if and only if there exist $\rho>0$ and $\bar{x} \in C$ such that $\sup _{t \in T}\left\{f_{t}, \delta_{C}-1\right\}(\bar{x})<-\rho$ if and only if $(i v)$ is true.

This concludes the proof.

We have shown that statements $(i)-(v i)$ in Theorem 5.1 are equivalent in $\Theta_{3}$ whereas $(i)-(v i i i)$ are equivalent in $\Theta_{5}$. The next three simple examples (with $|T|=1$ ) show that none of the properties defining $\Theta_{3}$ and $\Theta_{5}$ are superfluous. There, statements (ii)-(iv), (vii), and (viii) hold whereas $(i),(v)$ and $(v i)$ fail.

Example 5.2. Let $\sigma=\{f ; C\} \in \Theta_{2} \backslash\left(\Theta_{3} \cup \Theta_{5}\right)$ be such that $f(x)=x^{2}-1$ and $C=\{-1,0,1\} \subset \mathbb{R}(C$ is not convex $)$. We have $F=C, F_{S S}=\{0\}, f^{*}(v)=\frac{v^{2}}{4}+1$, and $\delta_{C}^{*}(v)=|v|$. Then

$$
\operatorname{clconv}\left(\operatorname{epi} f^{*}+\operatorname{epi} \delta_{C}^{*}\right)=\operatorname{clconv}\left(\operatorname{epi} f^{*} \cup\left[\operatorname{epi} \delta_{C}^{*}+(0,1)\right]\right)=\operatorname{epi} h,
$$

where $h(v)=\frac{v^{2}}{4}+1$, if $v \in[-2,2]$, and $h(v)=|v|$, otherwise.

EXAMPLE 5.3. Let $\sigma=\{f ; C\}$ be such that $f(x)=1-x^{2}$ and $C=[-1,+\infty[\subset \mathbb{R}$; $\sigma \in \Theta_{2} \backslash\left(\Theta_{3} \cup \Theta_{5}\right)$ because $F=\{-1\} \cup[1,+\infty[\nsubseteq \operatorname{int} C$ and $f$ is nonconvex. We have $\left.F_{S S}=\right] 1,+\infty\left[, f^{*}=+\infty\right.$, and $\delta_{C}^{*}(v)=-v$, if $v \leq 0$, and $\delta_{C}^{*}(v)=+\infty$, otherwise. On the other hand, taking, for $n \in \mathbb{N}, \sigma_{n}=\left\{f+\frac{1}{n} ; C\right\} \in \Theta_{2}$, we have $\mathcal{F}_{2}\left(\sigma_{n}\right)=\left[\sqrt{\frac{n+1}{n}},+\infty[\right.$, and this sequence converges to $[1,+\infty[$. Observe that here the set of (vii) is empty. 
Example 5.4. Consider $X=\mathbb{R}$ and the functions defined in Example 3.6. Let $\sigma=\{f-1 ; \mathbb{R}\} \in \Theta_{2} \backslash\left(\Theta_{3} \cup \Theta_{5}\right)$ (because the local-global property fails) and $\sigma_{n}=\left\{f_{n}-1 ; \mathbb{R}\right\} \subset \Theta_{3}$. It is easy to see that $d\left(\sigma_{n}, \sigma\right) \rightarrow 0, \mathcal{F}_{2}\left(\sigma_{n}\right)=[-1,1]$ for all $n \in \mathbb{N}$ and $\mathcal{F}_{2}(\sigma)=[-1,2]$. Finally, $\delta_{\mathbb{R}}^{*}=\delta_{\{0\}}$ and

$$
(f-1)^{*}(v)= \begin{cases}1, & \text { if } v \in\left[-1, \frac{1}{2}\right] \\ 2 v, & \text { if } \left.v \in] \frac{1}{2}, 1\right] \\ +\infty, & \text { otherwise }\end{cases}
$$

The latter example shows that, in contrast with convexity and extended convexity, continuity (or even smoothness) plays no role in the context of lower semicontinuity (consider, e.g., $\sigma=\{f ; \mathbb{R}\}$, with $f(x)=x(x-1)^{2}$ ).

REMARK 5.5. From the proof of $[(i v) \Rightarrow(i)]$, given $\hat{x} \in C$ such that $f_{t}(\hat{x}) \leq-\rho$ for all $t \in T$ (i.e., $\hat{x}$ is an SS-point of $\sigma$ with associated constant $\rho$ ), if $d\left(\sigma_{1}, \sigma\right)<$ $\min \left\{2^{1-k}, \rho, 2^{-1-k} \rho\right\}$, then $\hat{x}=x(1)$ is an SS-point of $\sigma_{1}$, with associated constant $\frac{\rho}{2}$.

Remark 5.6. If $\sigma=\left\{f_{t}, t \in T ; C\right\} \in \Theta_{6} \cap \operatorname{dom} \mathcal{F}$ and $C=X$ (e.g., $\sigma \in$ $\left.\Theta_{7} \cap \operatorname{dom} \mathcal{F}\right)$, then epi $\delta_{C}^{*}=$ cone $\{(0,1)\}$ and $f_{t}^{*}$ is a proper convex lsc function for all $t \in T$, so that epi $f_{t}^{*}+\operatorname{epi} \delta_{C}^{*}=\operatorname{epi} f_{t}^{*}$ for all $t \in T$. Thus

$$
\operatorname{cl} \operatorname{conv}\left\{\left(\bigcup_{t \in T} \operatorname{epi} f_{t}^{*}\right)+\operatorname{epi} \delta_{C}^{*}\right\} \subset \operatorname{cl} \operatorname{conv}\left\{\left(\bigcup_{t \in T} \operatorname{epi} f_{t}^{*}\right) \cup\left[\operatorname{epi} \delta_{C}^{*}+(0,1)\right]\right\},
$$

i.e., condition (vii) in Theorem 5.1 is weaker than condition (viii), and both conditions are equivalent whenever -1 is a lower bound for some constraint function $f_{t}$ (in which case $(0,1) \in$ epi $f_{t}^{*}$ and both sets in (5.8) coincide). The next example shows that the sets in (5.8) are generally different.

ExAmple 5.7. Let $X=\mathbb{R}$, and $\sigma=\{f ; \mathbb{R}\} \in \Theta_{5} \cap \operatorname{dom} \mathcal{F}$, with $f(x)=x+1$, if $x \leq 0$ and $f(x)=+\infty$, otherwise. Since $\delta_{C}^{*}=\delta_{\{0\}}$, and $f^{*}(v)=-1$, if $v \geq 1$, and $+\infty$, otherwise, we have

$$
\operatorname{clconv}\left\{\text { epi } f^{*}+\operatorname{epi} \delta_{C}^{*}\right\}=(1,-1)+\mathbb{R}_{+}^{2}
$$

and

$$
\operatorname{cl} \operatorname{conv}\left\{\operatorname{epi} f^{*} \cup\left[\operatorname{epi} \delta_{C}^{*}+(0,1)\right]\right\}=\operatorname{conv}\{(1,-1),(0,1)\}+\mathbb{R}_{+}^{2} .
$$

6. Robinson regularity. We can aggregate another characterization of the lsc property of $\mathcal{F}$ to the list in Theorem 5.1 when $X$ is a normable space and $B$ is a bounded barrel. Then the gauge associated with $B$, i.e.,

$$
\rho_{B}(x):=\inf \{\eta>0: x \in \eta B\}
$$

is actually a norm, the $\rho_{B}$-topology is exactly the original topology in $X[25, \S 10 \mathrm{C}]$, and the sets $\left\{\frac{1}{n} B: n=1,2, \ldots\right\}$ form a local base in $X$. We shall represent by $\delta_{B}$ the distance associated with $\rho_{B}$. Obviously, if $X$ is a normed space and $B$ is the closed unit ball, then $\delta_{B}$ is the distance associated with this norm. The next definition is inspired in [46]. 
Let $T \neq \emptyset$. The feasible set mapping $\mathcal{F}: \Theta \rightrightarrows X$ is said to be Robinson regular at $\sigma=\left\{f_{t}, t \in T ; C\right\} \in \Theta$ if for each $\widetilde{x} \in \mathcal{F}(\sigma)$, there exist $\epsilon, \beta>0$ such that $\widetilde{x} \in C_{1}$ and

$$
\delta_{B}\left(\widetilde{x}, \mathcal{F}\left(\sigma_{1}\right)\right) \leq \beta \max \left\{0, g^{1}(\widetilde{x})\right\},
$$

for any $\sigma_{1} \in \Theta$ such that $d\left(\sigma, \sigma_{1}\right)<\epsilon\left(g^{1}\right.$ denotes the marginal function of $\left.\sigma_{1}\right)$. Here, by convention, we understand that $\delta_{B}(\tilde{x}, \emptyset)=+\infty$.

Theorem 6.1. Let $X$ be a normable space, $B$ be a bounded barrel, and let $\sigma=\left\{f_{t}, t \in T ; C\right\} \in \operatorname{dom} \mathcal{F}$, with $T \neq \emptyset$. If $\mathcal{F}$ is Robinson regular at $\sigma \in \Theta_{3} \cup \Theta_{5}$, then $\mathcal{F}$ is lsc at $\sigma$. The converse statement holds if $\sigma \in \Theta_{5}$.

Proof. Suppose that $\mathcal{F}$ is Robinson regular at $\sigma \in \Theta_{3} \cup \Theta_{5}$. Take any $\widetilde{x} \in F$. Then there are real numbers $\epsilon, \beta>0$ such that for any $\sigma_{1}$ with $d\left(\sigma, \sigma_{1}\right)<\epsilon$, one has $\widetilde{x} \in C_{1}$ and

$$
\delta_{B}\left(\widetilde{x}, \mathcal{F}\left(\sigma_{1}\right)\right) \leq \beta \max \left[0, g^{1}(\widetilde{x})\right] .
$$

Let $\widetilde{k}$ be an integer number such that $\widetilde{x} \in \widetilde{k} B$. If $d\left(\sigma_{1}, \sigma\right)<2^{-\widetilde{k}} \epsilon$ then $d_{\widetilde{k}}\left(f_{t}, f_{t}^{1}\right)<$ $\epsilon$. In particular, we have

$$
f_{t}^{1}(\widetilde{x}) \leq \epsilon+f_{t}(\widetilde{x}) \leq \epsilon, \forall t \in T .
$$

This and (6.1) show that $\delta_{B}\left(\widetilde{x}, \mathcal{F}\left(\sigma_{1}\right)\right)<+\infty$, which implies $\mathcal{F}\left(\sigma_{1}\right) \neq \emptyset$ and hence, the statement (ii) in Theorem 5.1 holds. The lower semicontinuity of $\mathcal{F}$ follows from this theorem.

Now we assume that $\sigma \in \Theta_{5}$.

Assume that $\mathcal{F}$ is lsc at $\sigma$. By Theorem 5.1 (ii), $F_{1}:=\mathcal{F}\left(\sigma_{1}\right) \neq \emptyset$ if $\sigma_{1}=\left\{f_{t}^{1}, t \in\right.$ $\left.T ; C_{1}\right\} \in \Theta$ is close enough to $\sigma$.

Let $\widetilde{x} \in F=\mathcal{F}_{5}(\sigma)$. If $\delta_{B}\left(\widetilde{x}, F_{1}\right)=0$ then the conclusion holds trivially. Assume then that $\delta_{B}\left(\widetilde{x}, F_{1}\right)>0$, and let $r:=\delta_{B}\left(\widetilde{x}, F_{1}\right)$. Since $\left\{\frac{1}{n} B: n=1,2, \ldots\right\}$ is a local base in $X$, there exists $\eta^{\prime}>0$ such that $\left(\widetilde{x}+r \eta^{\prime} B\right) \cap F_{1}=\emptyset$.

By the separation theorem, there exists $v \in X^{*} \backslash\{0\}$ such that

$$
v(x) \leq v\left(\widetilde{x}-r \eta^{\prime} z\right)=v(\widetilde{x})-r \eta^{\prime} v(z), \forall x \in F_{1}, \forall z \in B .
$$

This leads us to

$$
v(x) \leq \inf _{z \in B}\left[v(\widetilde{x})-r \eta^{\prime} v(z)\right]=v(\widetilde{x})-r \eta, \forall x \in F_{1},
$$

where $\eta:=\eta^{\prime} \sup _{z \in B} v(z)$ is a nonnegative real number because $B$ is bounded, $0 \in B$, and $\eta^{\prime}>0$. From (2.2) we get

$$
(v, v(\widetilde{x})-r \eta) \in \operatorname{cl} \text { cone }\left\{\left(\bigcup_{t \in T} \operatorname{epi}\left(f_{t}^{1}\right)^{*}\right)+\operatorname{epi} \delta_{C_{1}}^{*}\right\} .
$$

Then there exist nets $\left\{\lambda^{\alpha}\right\}_{\alpha \in \Delta} \subset \mathbb{R}_{+}^{(T)},\left\{u_{t}^{\alpha}\right\}_{\alpha \in \Delta} \subset \operatorname{dom}\left(f_{t}^{1}\right)^{*},\left\{w^{\alpha}\right\}_{\alpha \in \Delta} \subset \operatorname{dom} \delta_{C_{1}}^{*}$ and $\left\{\beta^{\alpha}\right\}_{\alpha \in \Delta} \subset \mathbb{R}_{+}, t \in T$ such that

$$
\begin{gathered}
\lim _{\alpha}\left(\sum_{t \in T} \lambda_{t}^{\alpha} u_{t}^{\alpha}(x)+w^{\alpha}(x)\right)=v(x), \forall x \in X \\
\lim _{\alpha}\left(\sum_{t \in T} \lambda_{t}^{\alpha}\left(f_{t}^{1}\right)^{*}\left(u_{t}^{\alpha}\right)+\delta_{C_{1}}^{*}\left(w^{\alpha}\right)+\beta^{\alpha}\right)=v(\widetilde{x})-r \eta .
\end{gathered}
$$


Therefore,

$$
\begin{gathered}
\lim _{\alpha}\left\{\sum_{t \in T} \lambda_{t}^{\alpha}\left[u_{t}^{\alpha}(x)-\left(f_{t}^{1}\right)^{*}\left(u_{t}^{\alpha}\right)\right]+w^{\alpha}(x)-\delta_{C_{1}}^{*}\left(w^{\alpha}\right)-\beta^{\alpha}\right\} \\
=v(x)-v(\widetilde{x})+r \eta, \forall x \in X
\end{gathered}
$$

Note that for each $\alpha \in \Delta, t \in T$, and each $x \in X$,

$$
f_{t}^{1}(x)=\left(f_{t}^{1}\right)^{* *}(x) \geq u_{t}^{\alpha}(x)-\left(f_{t}^{1}\right)^{*}\left(u_{t}^{\alpha}\right),
$$

and if $x \in C_{1}$ then $w^{\alpha}(x)-\delta_{C_{1}}^{*}\left(w^{\alpha}\right) \leq 0$. We now can derive from (6.2) that

$$
v(x)-v(\widetilde{x})+r \eta \leq \bar{\lambda} \sup _{t \in T} f_{t}^{1}(x), \forall x \in C_{1},
$$

where $\bar{\lambda}:=\lim \sup \sum_{t \in T} \lambda_{t}^{\alpha}, \bar{\lambda} \in \mathbb{R} \cup\{+\infty\}$.

We will see that $\bar{\lambda}$ is finite. Indeed, if $\bar{x}$ is an SS-point of $\sigma$ (it exists by Theorem 5.1 (iv)) associated with $\rho>0$ then for $\sigma_{1}$ closed enough to $\sigma, \bar{x}$ is also an SS-point of $\sigma_{1}$, associated with $\frac{\rho}{2}$ (recall Remark 5.5), i.e., $\bar{x} \in C_{1}$ and $f_{t}^{1}(\bar{x}) \leq-\frac{\rho}{2}$ for all $t \in T$. Letting $x=\bar{x}$ in (6.3), we get

$$
v(\bar{x})-v(\widetilde{x}) \leq v(\bar{x})-v(\widetilde{x})+r \eta \leq \bar{\lambda}\left(-\frac{\rho}{2}\right),
$$

which implies that $\bar{\lambda}<+\infty$ and

$$
\bar{\lambda} \leq \frac{2}{\rho} v(\widetilde{x}-\bar{x}) \leq \frac{2}{\rho}|v(\widetilde{x})-v(\bar{x})| .
$$

We now observe that by the same argument as in Example 3.8 (a), $\widetilde{x} \in C_{1}$ whenever $\sigma_{1}$ is close enough to $\sigma$. So letting $x=\widetilde{x}$ in (6.3), we get

$$
r \eta \leq \bar{\lambda} g^{1}(\widetilde{x})
$$

which, together with (6.4), gives

$$
\delta_{B}\left(\widetilde{x}, F_{1}\right)=r \leq \beta g^{1}(\widetilde{x}),
$$

where $\beta:=\frac{2}{\rho \eta}|v(\widetilde{x})-v(\bar{x})|$. The conclusion follows.

ExAmple 6.2. Let $X=\mathbb{R}, B=[-1,1], f(x)=-x^{2}$, and $\sigma=\{f ; \mathbb{R}\} \in \Theta_{3}$. Assume that $\mathcal{F}_{3}$ is Robinson regular at $\sigma$, with constant $\beta>0$. Take $\widetilde{x}=0 \in F$ and the sequence $\left\{\sigma_{n}\right\}_{n \in \mathbb{N}}$ such that $\sigma_{n}=\left\{f_{n} ; \mathbb{R}\right\} \in \Theta_{3}$, with $f_{n}=f+\frac{1}{n}, n \in \mathbb{N}$. Then we have $\left.d\left(\sigma_{n}, \sigma\right) \rightarrow 0, \mathcal{F}_{3}\left(\sigma_{n}\right)=\right]-\infty,-\frac{1}{\sqrt{n}}[\cup] \frac{1}{\sqrt{n}},+\infty\left[, \delta_{B}\left(\widetilde{x}, \mathcal{F}_{3}\left(\sigma_{n}\right)\right)=\frac{1}{\sqrt{n}}\right.$ and $\max \left\{0, g^{n}(\widetilde{x})\right\}=\frac{1}{n}, n \in \mathbb{N}$. Since $\frac{1}{\sqrt{n}} \leq \frac{\beta}{n}$ for $n$ large enough, multiplying by $n$ both members of this inequality and taking limits as $n \rightarrow \infty$ we get a contradiction. Hence $\mathcal{F}_{3}$ is lsc (because $F_{S S}=\mathbb{R} \backslash\{0\}$ ) but it is not Robinson regular at $\sigma$.

7. Upper semicontinuity. In this section we give sufficient conditions for the usc property of the feasible set map at a consistent system $\sigma$ under perturbations of all the data. Obviously, these conditions are also sufficient in the case that we restrict ourselves to perturbations of the RHS function. We also characterize the usc property (Theorems 7.4 and 7.9) but, in contrast with the lsc counterpart (Theorem 5.1), such 
characterization, which also remains valid for RHS perturbations, does not involve the data. Also in contrast with the lsc property, $T=\emptyset$ does not imply the upper semicontinuity of the feasible set mapping everywhere.

Example 7.1. Let $\mathbb{B}$ be the closed unit ball in $X=\mathbb{R}^{2}, C=$ epi $x_{1}^{2}$ and $C_{n}=$ epi $h_{n}$, where

$$
h_{n}\left(x_{1}\right)= \begin{cases}-2 n x_{1}-n^{2}, & \text { if } x_{1} \leq-n, \\ x_{1}^{2}, & \text { if } \left.x_{1} \in\right]-n, n[, \\ 2 n x_{1}-n^{2}, & \text { if } x_{1} \geq n,\end{cases}
$$

$n \in \mathbb{N}$. Then $C_{n} \nsubseteq \Phi:=C+\mathbb{B}$ for all $n \in \mathbb{N}$, with $C \subset W$. Since $C_{n} \cap(k \mathbb{B})=C \cap(k \mathbb{B})$ for all $n \geq k, d\left(\delta_{C_{n}}, \delta_{C}\right) \rightarrow 0$ as $n \rightarrow \infty$ (by Corollary 3.4), so that $\mathcal{F}_{j}$ is not usc at $\sigma=\{x \in C\}, j=1, \ldots ., 7$ (in short, $j \geq 1$ ).

We first establish the usc property of $\mathcal{F} \cap K$ when $K$ is a compact set of $X$ at every consistent system. The next lemma can be seen as a local counterpart of the Closed Graph Theorem in [1, Theorem 17.11], which proves the equivalence between the closedness of the graph and the upper semicontinuity everywhere.

Lemma 7.2. If $K$ is a compact subset of $X$ then the truncated map $\widetilde{\mathcal{F}}: \Theta \rightrightarrows X$, $\Theta \subset \Theta_{2}$, defined by

$$
\widetilde{\mathcal{F}}\left(\sigma_{1}\right):=(\mathcal{F} \cap K)\left(\sigma_{1}\right)=\mathcal{F}\left(\sigma_{1}\right) \cap K \text { for all } \sigma_{1} \in \Theta,
$$

is usc at any $\sigma \in \operatorname{dom} \mathcal{F}$.

Proof. Let $\sigma \in \operatorname{dom} \mathcal{F}$. We observe firstly that the closedness of $\mathcal{F}$ at $\sigma$ entails the closedness of $\widetilde{\mathcal{F}}$ at this parameter.

Assume that $\widetilde{\mathcal{F}}$ is not usc at $\sigma$. Then there is an open set $W$ such that $\widetilde{\mathcal{F}}(\sigma) \subset W$ and for any neighborhood $V$ of $\sigma$ there exists $\sigma_{V} \in V$ and $x_{V} \in \widetilde{\mathcal{F}}\left(\sigma_{V}\right)$ with $x_{V} \notin W$. Note that $\left\{x_{V}\right\}$ is a net in $K \backslash W$ directed by inclusion (we are using the axiom of choice). Since $K$ is compact, there is a subnet of $\left\{x_{V}\right\}$ converging to a point in $K$, say $x \in K$. Then $x \in K \backslash W$ since this set is relatively closed in $K$, but then $x \notin \widetilde{\mathcal{F}}(\sigma)$, which contradicts the closedness of $\widetilde{\mathcal{F}}$ at $\sigma$. Consequently, $\widetilde{\mathcal{F}}$ is usc at $\sigma$.

Proposition 7.3. If $\Theta \subset \Theta_{6}$ and $C_{0}$ is compact, then $\mathcal{F}$ is usc at any $\sigma \in \operatorname{dom} \mathcal{F}$.

Proof. It is straightforward consequence of Lemma 7.2 taking $K=C_{0}$.

According to Proposition 7.5(ii) below, the boundedness of $\mathcal{F}(\sigma)$ entails the upper semicontinuity of $\mathcal{F}$ at $\sigma$ when $X=\mathbb{R}^{n}$ and $\Theta \subset \Theta_{6}$, but this property is seriously troublesome when $X$ is an infinite-dimensional normed space (where boundedness of $\mathcal{F}(\sigma)$ does not imply upper semicontinuity of $\mathcal{F}$ at $\sigma$ any longer [42, Example 3]).

7.1. Upper semicontinuity in $\mathbb{R}^{n}$. The next result characterizes the usc property of $\mathcal{F}$ provided the decision space $X$ is locally compact, in which case $X$ is finite dimensional and, so, isomorphic to $\mathbb{R}^{n}([34, \S 15.7(1)])$. The sufficient part is totally general and its proof is a straightforward consequence of statement (iii) in [22, Lemma 2 ]. For $j=6,7$, this result generalizes the corresponding ones in [21] and [44] because we consider here a fixed set constraint $C_{0} \subseteq \mathbb{R}^{n}$ and extended constraint functions $f_{t}$, $t \in T$.

Theorem 7.4. Let $\sigma \in \operatorname{dom} \mathcal{F}, \Theta \subset \Theta_{2}$. Then $\mathcal{F}$ is usc at $\sigma$ if and only if there exists a compact set $K$ and a positive number $\epsilon$ such that

$$
\mathcal{F}\left(\sigma_{1}\right) \backslash K \subset \mathcal{F}(\sigma) \backslash K \text { for all } \sigma_{1} \in \Theta \text { with } d\left(\sigma, \sigma_{1}\right)<\epsilon \text {. }
$$


In the present setting (i.e., $X=\mathbb{R}^{n}$ ) the usc property of $\mathcal{F}$ for $\Theta \subset \Theta_{5}$ follows from the boundedness of $\mathcal{F}(\sigma)$ as claimed in the next proposition, whose proof goes along the ways as those of [44, Lemma 4.3, Proposition 4.2] with a minor modification to treat with the lsc property of the functions and the set constraints involved.

Proposition 7.5. Let $\sigma \in \operatorname{dom} \mathcal{F}, \Theta \subset \Theta_{5}$. If $\mathcal{F}(\sigma)$ is bounded then the following statements hold:

(i) $\mathcal{F}$ is uniformly bounded in some neighborhood of $\sigma$.

(ii) $\mathcal{F}$ is usc at $\sigma$.

Proof. Let $\sigma \in \operatorname{dom} \mathcal{F}, \Theta \subset \Theta_{5}$. We assume $T \neq \emptyset$. We need to prove (i) only since (ii) follows immediately from (i).

Let $\mathbb{B}$ be the closed unit ball in $\mathbb{R}^{n}$. Suppose that $F:=\mathcal{F}(\sigma) \subset \mu \mathbb{B}$ for some $\mu>0$. Assume that (i) does not hold. Then, there exist sequences $\left\{\sigma_{r}\right\} \subset \Theta$ and $\left\{z_{r}\right\} \subset \mathbb{R}^{n}$ such that $d\left(\sigma, \sigma_{r}\right)<\frac{1}{r}, z_{r} \in F_{r}:=\mathcal{F}\left(\sigma_{r}\right)$ with $\left\|z_{r}\right\|>r$ for all $r \in \mathbb{N}$.

Now let $\lambda_{r}=\frac{3 \mu}{\left\|z_{r}\right\|}$. Then with $r>3 \mu, 0<\lambda_{r}<1$ and $\lambda_{r} \rightarrow 0$ as $r \rightarrow \infty$. Furthermore, without loss of generality, we can assume that $\lambda_{r} z_{r} \rightarrow z$ as $r \rightarrow \infty$ with $\|z\|=3 \mu$.

We now take $\bar{x} \in F$ and set $w_{r}:=\lambda_{r} z_{r}+\left(1-\lambda_{r}\right) \bar{x}$. It turns out that $w_{r} \rightarrow z+\bar{x}$ as $r \rightarrow \infty$.

Fix $t \in T$. Then, for each $r$, the convexity of $f_{t}^{r}$ gives

$$
f_{t}^{r}\left(w_{r}\right) \leq \lambda_{r} f_{t}^{r}\left(z_{r}\right)+\left(1-\lambda_{r}\right) f_{t}^{r}(\bar{x}) \leq\left(1-\lambda_{r}\right) f_{t}^{r}(\bar{x}) .
$$

Now for any $\epsilon>0$, by the lsc of $f_{t}$, and by the convergence of the sequence $\left\{w_{r}\right\}$, there exist a relative compact neighborhood $V$ of $z+\bar{x}$ and an integer $r_{0}$ such that $w_{r} \in V$ and such that

$$
f_{t}(z+\bar{x})-f_{t}\left(w_{r}\right)<\epsilon
$$

for all $r \geq r_{0}$. On the other hand, since $d\left(\sigma, \sigma_{r}\right) \rightarrow 0$, it follows from the definition of this convergence and (7.5) that there is a compact set $B_{r^{\prime}}$ such that $\operatorname{cl} V \subset B_{r^{\prime}}$ and the sequence $\left\{f_{t}^{r}\right\}$ converges uniformly to $f_{t}$ on $B_{r^{\prime}}$, which entails that there is an integer $r_{1} \geq r_{0}$ such that

$$
f_{t}\left(w_{r}\right)-f_{t}^{r}\left(w_{r}\right)<\epsilon \text { for all } r \geq r_{1} .
$$

Therefore, if $r>\max \left\{r_{1}, 3 \mu\right\}$ then, combining (7.2) and (7.3), we get

$$
f_{t}(z+\bar{x})-f_{t}^{r}\left(w_{r}\right)=f_{t}(z+\bar{x})-f_{t}\left(w_{r}\right)+f_{t}\left(w_{r}\right)-f_{t}^{r}\left(w_{r}\right)<2 \epsilon,
$$

or equivalently,

$$
f_{t}(z+\bar{x}) \leq f_{t}^{r}\left(w_{r}\right)+2 \epsilon .
$$

This, together with (7.1) gives

$$
f_{t}(z+\bar{x}) \leq\left(1-\lambda_{r}\right) f_{t}^{r}(\bar{x})+2 \epsilon,
$$

which, in turn, gives rise to $f_{t}(z+\bar{x}) \leq f_{t}(\bar{x})+2 \epsilon \leq 2 \epsilon$ since the sequence $\left\{f_{t}^{r}\right\}$ converges to $f_{t}, \lambda_{r} \rightarrow 0$ as $r \rightarrow \infty$, and $\bar{x} \in F$.

Consequently, we get $f_{t}(z+\bar{x}) \leq 0$ for any $t \in T$ since the last inequality holds for any $\epsilon>0$ and for any $t \in T$. 
The same argument applies to the sequence $\left\{\delta_{C_{r}}\right\}$. Thus we also get $\delta_{C}(z+\bar{x}) \leq 0$, which shows that $z+\bar{x} \in C$. So, $z+\bar{x} \in F$, but $\|z+\bar{x}\| \geq\|z\|-\|\bar{x}\| \geq 2 \mu$, which contradicts the fact that $F \subset \mu \mathbb{B}$. Finally, we may conclude that there exists some $\bar{r}>0$ such that $F_{1}=\mathcal{F}\left(\sigma_{1}\right) \subset \bar{r} \mathbb{B}$ for any $\sigma_{1} \in \Theta$ satisfying $d\left(\sigma, \sigma_{1}\right)<\frac{1}{\bar{r}}$.

The next example shows that both statements in Proposition 7.5 fail for $j=3$.

ExAmPle 7.6. Let $\sigma=\{f ; \mathbb{R}\}$, with $f(x)=x^{2} \exp (x)$ and $\sigma_{k}=\left\{f_{k} ; \mathbb{R}\right\}$ such that $f_{k}=f-\frac{1}{k} \in \mathcal{V}_{3}, k \in \mathbb{N}$. All these systems are in $\Theta_{3}$ because the unique local minimum of their constraint function, 0 , is global. Let $x_{r}<y_{r}<z_{r}$ be the three real roots of $f(x)=\frac{1}{r}$, with $x_{r} \rightarrow \infty$ and $y_{r}, z_{r} \rightarrow 0$. We have $d\left(\sigma_{r}, \sigma\right) \rightarrow 0, \mathcal{F}_{3}(\sigma)=\{0\}$, and $\left.\left.\mathcal{F}_{3}\left(\sigma_{r}\right)=\right]-\infty, x_{r}\right] \cup\left[y_{r}, z_{r}\right], r \in \mathbb{N}$. Thus $\mathcal{F}_{3}$ is neither uniformly bounded nor usc at $\sigma$ although $\mathcal{F}_{3}(\sigma)$ is compact.

In the following corollary we provide a sufficient condition for the upper semicontinuity of $\mathcal{F}_{j}$, for $j \geq 5$, which relies on the nominal data.

Corollary 7.7. Let $\sigma \in \operatorname{dom} \mathcal{F}, \Theta \subset \Theta_{5}$. Then, the following conditions are both equivalent to the boundedness of $\mathcal{F}(\sigma)$ and, so, they imply the upper semicontinuity of $\mathcal{F}$ at $\sigma$ :

(i) $(0,1) \in \operatorname{int}$ cone $\left\{\left(\bigcup_{t \in T} \operatorname{epi} f_{t}^{*}\right) \cup\left[\operatorname{epi} \delta_{C}^{*}+(0,1)\right]\right\}$.

(ii) The projection of the cone above on the space of the first $n$ coordinates is $\mathbb{R}^{n}$.

Proof. It is a straightforward consequence of [19, Theorem 9.3].

In [11] a characterization of the upper semicontinuity of $\mathcal{F}_{7}$ is given in terms of the so-called reinforced system associated with the nominal system $\sigma$, although checking this condition is rather difficult. In [8] another characterization of the upper semicontinuity of $\mathcal{F}$ is provided in the particular setting of continuous linear semiinfinite programming, and this conditions is that $\mathcal{F}(\sigma)$ is either bounded or the whole space $\mathbb{R}^{n}$.

7.2. Upper semicontinuity in locally compact sigma-compact spaces. Let $X$ be a locally compact topological space (without linear structure, noncompact). We also assume that $X$ is sigma-compact; i.e. (see [34, page 22]), there exists a sequence $\left\{K_{r}\right\}_{r \in \mathbb{N}}$ of compact sets such that

$$
K_{r} \subset \operatorname{int} K_{r+1}, \forall r \in \mathbb{N}, X=\bigcup_{r=1}^{\infty} K_{r}=\bigcup_{r=1}^{\infty} \operatorname{int} K_{r} .
$$

Moreover,

$$
\text { For all compact set } K \subset X, \exists r_{0} \text { such that } K \subset K_{r_{0}} \text {. }
$$

Consider the space of all real-valued continuous functions on $X, \mathcal{C}(X)$, endowed with the uniformly convergent topology, $\tau$, on compact subsets of $X$. A base of $\tau$ neighborhoods of 0 consists of the set of functions $f$ such that $\sup _{x \in K}|f(x)|<\epsilon$, where $\epsilon>0$ and $K$ is a compact subset of $X$. Moreover, $\mathcal{C}(X)$ is a locally convex, complete space with the topology defined by all the seminorms $p_{K}(f):=\sup _{x \in K}|f(x)|$, where $K$ is a compact subset of $X$.

Under the assumptions, $\mathcal{C}(X)$ is a Fréchet space (i.e., locally convex, complete, and metrizable). Moreover, its topology is defined by countably many seminorms $p_{K_{r}}$ 
([34, page 250]). But then the metric that generates the topology in $\mathcal{C}(X)$ can be defined by

$$
\rho(f, h)=\sum_{r=1}^{\infty} 2^{-r} \min \left\{1, p_{K_{r}}(f-h)\right\}, f, h \in \mathcal{C}(X)
$$

(compare with the metric $d$ defined in $\mathcal{V}_{1}$ in $\S 3$ ). It is easy to see that the conclusions of technical Lemmas 3.1 and 3.2 in $\S 3$ still hold (with the same proof) for the metric $\rho$ on $\mathcal{C}(X)$, with $d_{r}$ replaced here by $p_{K_{r}}$.

Let $T$ be an arbitrary index set as before and let

$$
\Theta_{8}:=\left\{\left\{f_{t}^{1}, t \in T ; X\right\}: f_{t}^{1} \in \mathcal{C}(X) \forall t \in T\right\} .
$$

Given $\sigma=\left\{f_{t}, t \in T ; X\right\}, \sigma_{1}=\left\{f_{t}^{1}, t \in T ; X\right\} \in \Theta_{8}$, we define

$$
d\left(\sigma, \sigma_{1}\right):=\sup _{t \in T} \rho\left(f_{t}, f_{t}^{1}\right) .
$$

If $\Theta \subset \Theta_{8}$, then $(\Theta, d)$ is a metric space, which is complete when $\Theta$ is closed in $\Theta_{8}$ (same proof as in Proposition 3.7). We are now in a position to establish the closedness and the upper semicontinuity of the feasible set mapping $\mathcal{F}$. The proof of the next result is similar to those of Theorem 4.1 and Proposition 4.2 and will be omitted.

Theorem 7.8. Let $\Theta \subset \Theta_{8}$. Then the following statements hold:

(i) $\mathcal{F}$ is closed.

(ii) If $\left\{\sigma_{\delta}\right\}_{\delta \in \Delta} \subset \operatorname{dom} \mathcal{F}$ and $\lim _{\delta} \sigma_{\delta}=\sigma$, then $L s_{\delta} \mathcal{F}\left(\sigma_{\delta}\right) \subset \mathcal{F}(\sigma)$.

Concerning the lsc property, we have seen that continuity is irrelevant, i.e., for the statements in Theorem 5.1, (i) $\Rightarrow(i i) \Rightarrow(i i i) \Rightarrow(i v)$ but $(i v) \nRightarrow(i)$ (recall Example $5.4)$.

Theorem 7.9. Let $\sigma \in \operatorname{dom} \mathcal{F}, \Theta \subset \Theta_{8}$, and let $X$ be metrizable. Then $\mathcal{F}$ is usc at $\sigma$ if and only if there exist a compact set $K$ and a positive number $\epsilon$ such that

$$
\mathcal{F}\left(\sigma_{1}\right) \backslash K \subset \mathcal{F}(\sigma) \backslash K \text { for all } \sigma_{1} \in \Theta \text { with } d\left(\sigma, \sigma_{1}\right)<\epsilon \text {. }
$$

Proof. For simplicity, we write in this proof $F_{r}:=\mathcal{F}\left(\sigma_{r}\right)$ for all $\sigma_{r} \in \Theta$.

Assume that (7.7) holds. Let $W$ be an open set containing $F$. Since $\mathcal{F}$ is closed at $\sigma$ (by Theorem 7.9, part (i)) and $K$ is compact, it follows from Lemma 7.2 that the map $\mathcal{F} \cap K$ is usc at $\sigma$. So, there will exist certain $\epsilon_{1}>0, \epsilon_{1}<\epsilon$, such that for each $\sigma_{1}$ satisfying $d\left(\sigma, \sigma_{1}\right)<\epsilon_{1}$, one has

$$
F_{1} \cap K \subset W .
$$

Now, by assumption $F_{1} \backslash K \subset F \backslash K$, so that

$$
\begin{aligned}
F_{1} & =\left(F_{1} \cap K\right) \cup\left(F_{1} \backslash K\right) \\
& \subset W \cup(F \backslash K) \subset W,
\end{aligned}
$$

which shows that $\mathcal{F}$ is usc at $\sigma$. 
Now we suppose that condition (7.7) is not satisfied. Let $\left\{K_{r}\right\}_{r \in \mathbb{N}}$ be a sequence of compact sets in $X$ satisfying (7.4). Then, there exist sequences $\left\{\sigma_{r}\right\}_{r \in \mathbb{N}} \subset \operatorname{dom} \mathcal{F}$ and $\left\{x_{r}\right\}_{r \in \mathbb{N}} \subset X$ satisfying

$$
\begin{aligned}
& d\left(\sigma, \sigma_{r}\right)<\frac{1}{r}, \\
& x_{r} \in F_{r} \backslash K_{r}, \\
& x_{r} \notin F \backslash K_{r}
\end{aligned}
$$

for all $r \in \mathbb{N}$. Thus, $\sigma_{r} \rightarrow \sigma$ and $x_{r} \notin K_{m}$ for every $r \geq m$ (since the sequence $\left\{K_{r}\right\}_{r \in \mathbb{N}}$ is nested). It is easy to see that such sequence $\left\{x_{r}\right\}_{r \in \mathbb{N}}$ has no accumulation point. In fact, if $x_{0}$ is an accumulation point of this sequence then $x_{0}$ must be contained in some $\operatorname{int} K_{r_{0}+1}$, which entails that int $K_{r_{0}+1}$ is a neighborhood of $x_{0}$ that contains only a finite number of elements of the sequence $\left\{x_{r}\right\}$. This is impossible.

Since $x_{r} \in F_{r} \backslash F, \sigma_{r} \rightarrow \sigma$, as $r \rightarrow \infty$, and $\left\{x_{r}\right\}_{r \in \mathbb{N}}$ has no accumulation point, $\mathcal{F}$ is not usc at $\sigma$ as a consequence of the Dolecki condition ([4, Lemma 2.2.2]). The proof is complete.

Acknowledgment. The authors wish to thank R.I. Bot for having proposed us the elegant proof of the equivalence $(i v) \Leftrightarrow(v i i) \Leftrightarrow(v i i i)$ in Theorem 5.1, P.P. Dong for his valuable suggestion concerning the proof of Theorem 6.1, J.E. Martínez-Legaz for suggesting the possible interest of $\Theta_{3}$ in stability theory, the two anonymous Referees whose valuable comments and suggestions have improved significantly the quality of the manuscript and, last but not least, the University of Alicante for providing financial support to the visits of N. Dinh in June 2007, July 2008 and July 2009.

\section{REFERENCES}

[1] Aliprantis, C.D., Borders, K.C., Infinite Dimensional Analysis, a Hitchhiker's Guide (3rd Edition), Springer-Verlag, Berlin, 2006.

[2] Amaya, J., Goberna, M.A., On the stability of linear systems with an exact constraint set, Math. Methods Oper. Res. 63 (2006) 107-121.

[3] Amaya, J., Bosch, P., Goberna, M.A., Stability of the feasible set mapping of linear systems with an exact constraint set, Set-Valued Anal. 16 (2008) 621-635.

[4] Bank, B., Guddat, J., Klatte, D., Kummer, B., Tammer, K., Non-Linear Parametric Optimization, Birkhäuser Verlag, Basel, 1983.

[5] Bonanno, G., On a class of functionals whose local minima are global, J. Global Optim. 12 (1998) 101-104.

[6] Boţ, R.I., Csetnek, E.R., Wanka, G., Regularity conditions via quasi-relative interior in convex programming, SIAM. J. Optim. 19 (2008) 217-233.

[7] Boț, R.I., Grad, S.-M., Wanka, G., Generalized Moreau-Rockafellar results for composed convex functions, Optimization, to appear.

[8] Brosowski, B., Parametric Semi-Infinite Optimization, Verlag Peter Lang, Frankfurt-am-Main, 1982.

[9] Cánovas, M.J., Dontchev, A.L., López, M.A., Parra, J., Metric regularity of semi-infinite constraint systems, Math. Programming 104B (2005) 329-346.

[10] Cánovas, M.J., Gómez-Senent, F., Parra, J., Regularity modulus of arbitrarily perturbed linear inequality systems, J. Math. Anal. Appl. 343 (2008) 315-327.

[11] Cánovas, M.J., López, M.A., Parra, J., Upper semicontinuity of the fesible set mapping for linear inequalities systems, Set-Valued Anal. 10 (2002) 361-378.

[12] Cánovas M.J., López M.A., Mordukhovich B., Parra J., Variational analysis in semi-infinite and infinite programming, II: Necessary optimality conditions, Preprint 2010.

[13] Dinh, N., Goberna, M.A., López, M.A., From linear to convex systems: consistency, Farkas Lemma and applications, J. Convex Analysis 13 (2006) 279-290.

[14] Dinh, N., Goberna, M.A., López, M.A., Son, T.Q., New Farkas-type constraint qualifications in convex infinite programming, ESAIM: Control, Optim. \& Calculus of Variations 13 (2007) 580-597. 
[15] Fang, D.H., Li, C., Ng, K.F., Constraint qualifications for extended Farkas's lemmas and Lagrangian dualities in convex infinite programming, SIAM J. Optim. 20 (2009) 13111332.

[16] Fisher, T., Contributions to semi-infinite linear optimization, in B. Brosowski and E. Martensen (eds.) Approximation and Optimization in Mathematical Physics, Peter Lang, Frankfurtam-Main, 1983, pp. 175-199.

[17] Gayá, V.E., López, M.A., Vera de Serio, V.N., Stability in convex semi-infinite programming and rates of convergence of optimal solutions of discretized finite subproblems, Optimization 52 (2003) 693-713.

[18] Goberna, M.A., Larriqueta, M., Vera de Serio, V.N., On the stability of the boundary of the feasible set in linear optimization, Set-Valued Anal. 11 (2003) 203-223.

[19] Goberna, M.A., López, M.A., Linear Semi-Infinite Optimization, Wiley, Chichester, U.K., 1998.

[20] Goberna, M.A., López, M.A, Todorov, M.I., Stability theory for linear inequality systems, SIAM J. Matrix Anal. Appl. 17 (1996) 730-743.

[21] Goberna, M.A., López, M.A, Todorov, M.I., Stability theory for linear inequality systems II: Upper semicontinuity of the solution set mapping, SIAM J. Optim. 7 (1997) 1138-1151.

[22] Goberna, M.A., López, M.A, Todorov, M.I., The stability of closed-convex-valued mappings and the associated boundaries, J. Math. Anal. Appl. 306 (2005) 502-515.

[23] Greenberg, H.J., Pierskalla, W.P., Stability theorems for infinitely constrained mathematical programs, J. Optim. Theory Appl. 16 (1975) 409-428.

[24] Hettich, R., Jongen, H.Th., Semi-infinite programming: conditions of optimality and applications, in J. Stoer (ed) Optimization techniques, part 2, Lecture Notes in Control and Information Sciences, vol. 7, Springer-Verlag, Berlin, 1978, pp. 1-11.

[25] Holmes, R.B., Geometric Functional Analysis and its Applications, Springer-Verlag, New York, 1975.

[26] Horst, R., Thach, P.T., A topological property of limes-arcwise strictly quasiconvex functions, J. Math. Anal. Appl. 134 (1988) 426-430.

[27] Ioffe, A.D., On robustness of the regularity property of maps, Control Cybernet. 32 (2003) 543-554.

[28] Jeyakumar, V., Infinite-dimensional convex programming with applications to constrained approximation, J. Optim. Theory Appl. 75 (1992), 569-586.

[29] Jeyakumar, V., Asymptotic dual conditions characterizing optimality for infinite convex programs, J. Optim. Theory Appl. 93 (1997) 153-165.

[30] Jongen, H.Th., Rückmann, J.J., On stability and deformation in semi-infinite optimization, in R. Reemtsen, J.J. Rückmann (eds) Semi-infinite programming, Kluwer, Boston, 1998, pp. $29-67$.

[31] Jongen, H.Th., Rückmann, J.J., Weber, G.H., One parametric semi-infinite optimization: on the stability of the feasible set, SIAM J. Optim. 4 (1994) 637-648.

[32] Jongen, H.Th., Twilt, F., Weber, G.H., Semi-infinite optimization: structure and stability of the feasible set, J. Optim. Theory Appl. 72 (1992) 529-552.

[33] Klatte, D., Henrion, R., Regularity and stability in nonlinear semi-infinite optimization, in R. Reemtsen, J.J. Rückmann (eds) Semi-infinite programming, Kluwer, Boston, 1998, pp. 69-102.

[34] Köthe, G., Topological Vector Spaces I, Springer-Verlag, Berlin, 1969.

[35] Laurent, P.-J., A pproximation et Optimisation, Hermann, Paris, 1972.

[36] Li, X.F., Dong, J.L., Subvexormal functions and subvex functions, J. Optim. Theory Appl. 103 (1999) 675-704.

[37] Li, C., Ng, K.F., Constraint qualification, the strong CHIP, and best approximation with convex constraints in Banach spaces, SIAM J. Optim. 14 (2003) 584-607.

[38] Li, C., Ng, K.F., Strong CHIP for infinite system of closed convex sets in normed linear spaces, SIAM J. Optim. 16 (2005) 311-340.

[39] Li, C., Ng, K.F., On constraint qualification for an infinite system of convex inequalities in a Banach space, SIAM J. Optim. 15 (2005) 488-512.

[40] Li, C., Ng, K.F., Pong, T.K., The SECQ, linear regularity, and the strong CHIP for an infinite system of closed convex sets in normed linear spaces, SIAM J. Optim. 18 (2007) 643-665.

[41] Li, C., Ng, K.F., Pong, T.K., Constraint qualifications for convex inequality systems with applications in constrained optimization, SIAM J. Optim. 19 (2008), 163-187.

[42] López, M.A., Mira, J.A., Torregrosa, G., On the stability of infinite-dimensional linear inequality systems, Numer, Funct. Anal. Optim. 19 (1998) 1065-1077.

[43] López, M.A., Rubinov, A., Vera de Serio, V., Stability of semi-infinite inequality systems involving min-type functions, Numer. Funct. Anal. Optim. 26 (2005) 81-112. 
[44] López, M.A., Vera de Serio, V., Stability of the feasible set mapping in convex semi-infinite programming, in M.A. Goberna, M.A. López (eds) Semi-infinite programming: Recent Advances, Kluwer, Dordrecht, 2001, pp. 101-120.

[45] Mordukhovich, B.S., Variational analysis and generalized differentiation, Springer-Verlag, Berlin, 2006.

[46] Robinson, S.M., Stability theory for systems of inequalities. I. Linear systems, SIAM J. Numer. Anal. 12 (1975) 754-769.

[47] Rockafellar, R., Wets, R.J.-B., Variational Analysis, Springer-Verlag, Berlin, 1998.

[48] Rubinov, A., Abstract Convexity and Global Optimization, Kluwer, Dordrecht, 2000.

[49] Tuy, H., Stability property of a system of inequalities, Math. Oper. Statist. Series Opt. 8 (1977) 27-39.

[50] Zang, I., Avriel, M., On functions whose local minima are global, J. Optim. Theory Appl. 16 (1975) 183-190. 